\title{
Blow-up analysis for approximate Dirac-harmonic maps in dimension 2 with applications to the Dirac-harmonic heat flow
}

\author{
Jürgen Jost ${ }^{1} \cdot$ Lei Liu' ${ }^{1} \cdot$ Miaomiao $\mathrm{Zhu}^{2}$
}

Received: 26 September 2016 / Accepted: 15 June 2017 / Published online: 10 July 2017

(C) The Author(s) 2017. This article is an open access publication

\begin{abstract}
Dirac-harmonic maps couple a second order harmonic map type system with a first nonlinear Dirac equation. We consider approximate Dirac-harmonic maps $\left\{\left(\phi_{n}, \psi_{n}\right)\right\}$, that is, maps that satisfy the Dirac-harmonic system up to controlled error terms. We show that such approximate Dirac-harmonic maps defined on a Riemann surface, that is, in dimension 2 , continue to satisfy the basic properties of blow-up analysis like the energy identity and the no neck property. The assumptions are such that they hold for solutions of the heat flow of Dirac-harmonic maps. That flow turns the harmonic map type system into a parabolic system, but simply keeps the Dirac equation as a nonlinear first order constraint along the flow. As a corollary of the main result of this paper, when such a flow blows up at infinite time at interior points, we obtain an energy identity and the no neck property.
\end{abstract}

Mathematics Subject Classification 53C43 (58E20)

Communicated by L. Ambrosio.

The research leading to these results is building upon work that has received funding from the European Research Council under the European Union's Seventh Framework Programe (FP7/2007-2013) / ERC Grant Agreement No. 267087. The third author was supported in part by National Natural Science Foundation of China (No. 11601325). The authors would like to thank the referee for detailed and useful comments.

Jürgen Jost

jost@mis.mpg.de

Lei Liu

leiliu@mis.mpg.de; llei1988@mail.ustc.edu.cn

Miaomiao Zhu

mizhu@sjtu.edu.cn

1 Max Planck Institute for Mathematics in the Sciences, Inselstrasse 22, 04103 Leipzig, Germany

2 School of Mathematical Sciences, Shanghai Jiao Tong University, 800 Dongchuan Road, Shanghai 200240, China 


\section{Introduction}

Dirac-harmonic maps were introduced and studied in [2,3]. They were motivated by the supersymmetric nonlinear sigma model from quantum field theory $[6,10]$, and they combine and generalize the theories of harmonic maps and harmonic spinors.

Let us recall the precise definiton. Let $M$ be a compact Riemann surface, equipped with a Riemannian metric $h$ and with a fixed spin structure, $\Sigma M$ be the spinor bundle over $M$ and $\langle\cdot, \cdot\rangle_{\Sigma M}$ be the metric on $\Sigma M$ induced by the Riemannian metric $h$. Choosing a local orthonormal basis $e_{\alpha}, \alpha=1,2$ on $M$, the usual Dirac operator is defined as $\not \partial:=e_{\alpha} \cdot \nabla_{e_{\alpha}}$, where $\nabla$ is the spin connection on $\Sigma M$. The usual Dirac operator $\not \partial$ on a surface can be seen as the Cauchy-Riemann operator. Consider $\mathbb{R}^{2}$ with the Euclidean metric $d x^{2}+d y^{2}$. Let $e_{1}=\frac{\partial}{\partial x}$ and $e_{2}=\frac{\partial}{\partial y}$ be the standard orthonormal frame. A spinor field is simply a map $\psi: \mathbb{R}^{2} \rightarrow \Delta_{2}=\mathbb{C}^{2}$, and the action of $e_{1}$ and $e_{2}$ on spinors can be identified with multiplication with matrices

$$
e_{1}=\left(\begin{array}{cc}
0 & 1 \\
-1 & 0
\end{array}\right), \quad e_{2}=\left(\begin{array}{cc}
0 & i \\
i & 0
\end{array}\right)
$$

If $\psi:=\left(\begin{array}{l}\psi_{1} \\ \psi_{2}\end{array}\right): \mathbb{R}^{2} \rightarrow \mathbb{C}^{2}$ is a spinor field, then the Dirac operator is

$$
\not \partial \psi=\left(\begin{array}{cc}
0 & 1 \\
-1 & 0
\end{array}\right)\left(\begin{array}{c}
\frac{\partial \psi_{1}}{\partial x} \\
\frac{\partial \psi_{2}}{\partial x}
\end{array}\right)+\left(\begin{array}{cc}
0 & i \\
i & 0
\end{array}\right)\left(\begin{array}{c}
\frac{\partial \psi_{1}}{\partial y} \\
\frac{\partial \psi_{2}}{\partial y}
\end{array}\right)=2\left(\begin{array}{c}
\frac{\partial \psi_{2}}{\partial \bar{z}} \\
-\frac{\partial \psi_{1}}{\partial z}
\end{array}\right),
$$

where

$$
\frac{\partial}{\partial z}=\frac{1}{2}\left(\frac{\partial}{\partial x}-i \frac{\partial}{\partial y}\right), \quad \frac{\partial}{\partial \bar{z}}=\frac{1}{2}\left(\frac{\partial}{\partial x}+i \frac{\partial}{\partial y}\right) .
$$

For more details on spin geometry and Dirac operators, one can refer to [14].

Let $\phi$ be a smooth map from $M$ to another compact Riemannian manifold $(N, g)$ with dimension $n \geq 2$. Let $\phi^{\star} T N$ be the pull-back bundle of $T N$ by $\phi$ and then we get the twisted bundle $\Sigma M \otimes \phi^{\star} T N$. Naturally, there is a metric $\langle\cdot, \cdot\rangle_{\Sigma M \otimes \phi^{\star} T N}$ on $\Sigma M \otimes \phi^{\star} T N$ which is induced from the metrics on $\Sigma M$ and $\phi^{\star} T N$. Also we have a natural connection $\widetilde{\nabla}$ on $\Sigma M \otimes \phi^{\star} T N$ which is induced from the connections on $\Sigma M$ and $\phi^{\star} T N$. Let $\psi$ be a section of the bundle $\Sigma M \otimes \phi^{\star} T N$. In local coordinates, it can be written as

$$
\psi=\psi^{i} \otimes \partial_{y^{i}}(\phi),
$$

where each $\psi^{i}$ is a usual spinor on $M$ and $\partial_{y^{i}}$ is the nature local basis on $N$. Then $\widetilde{\nabla}$ becomes

$$
\widetilde{\nabla} \psi=\nabla \psi^{i} \otimes \partial_{y^{i}}(\phi)+\left(\Gamma_{j k}^{i} \nabla \phi^{j}\right) \psi^{k} \otimes \partial_{y^{i}}(\phi),
$$

where $\Gamma_{j k}^{i}$ are the Christoffel symbols of the Levi-Civita connection of $N$. The Dirac operator along the map $\phi$ is defined by $\not D \psi:=e_{\alpha} \cdot \widetilde{\nabla}_{e_{\alpha}} \psi$.

We consider the following functional

$$
\begin{aligned}
L(\phi, \psi) & =\int_{M}\left(|d \phi|^{2}+\langle\psi, \not D \psi\rangle_{\Sigma M \otimes \phi^{\star} T N}\right) d M \\
& =\int_{M}\left(g_{i j}(\phi) h^{\alpha \beta} \frac{\partial \phi^{i}}{\partial x^{\alpha}} \frac{\partial \phi^{j}}{\partial x^{\beta}}+g_{i j}(\phi)\left\langle\psi^{i}, \not D \psi^{j}\right\rangle_{\Sigma M}\right) d M
\end{aligned}
$$


The functional $L(\phi, \psi)$ is conformally invariant. That is , for any conformal diffeomorphism $f: M \rightarrow M$, setting

$$
\widetilde{\phi}=\phi \circ f \quad \text { and } \quad \tilde{\psi}=\lambda^{-1 / 2} \psi \circ f .
$$

Then $L(\widetilde{\phi}, \widetilde{\psi})=L(\phi, \psi)$. For the proof, one can refer to [3]. Here $\lambda$ is the conformal factor of the conformal map f, i.e. $f^{*} h=\lambda^{2} h$. Critical points $(\phi, \psi)$ are called Dirac-harmonic maps from $M$ to $N$.

The Euler-Lagrange equations of the functional $L$ are

$$
\begin{gathered}
\left(\Delta \phi^{i}+\Gamma_{j k}^{i} h^{\alpha \beta} \phi_{\alpha}^{j} \phi_{\beta}^{k}\right) \frac{\partial}{\partial y^{i}}(\phi(x))=R(\phi, \psi), \\
\not D \psi=0,
\end{gathered}
$$

where $R(\phi, \psi)$ is defined by

$$
R(\phi, \psi)=\frac{1}{2} R_{l i j}^{m}(\phi(x))\left\langle\psi^{i}, \nabla \phi^{l} \cdot \psi^{j}\right\rangle \frac{\partial}{\partial y^{m}}(\phi(x)) .
$$

Here $R_{l i j}^{m}$ stands for the Riemann curvature tensor of the target manifold $(N, g)$. One can refer to $[2,3]$.

By the Nash embedding theorem, we embed $N$ into $\mathbb{R}^{K}$. Then, the critical points $(\phi, \psi)$ satisfy the Euler-Lagrange equations

$$
\begin{gathered}
\Delta \phi=A(\phi)(d \phi, d \phi)+\operatorname{Re}\left(P\left(\mathcal{A}\left(d \phi\left(e_{\alpha}\right), e_{\alpha} \cdot \psi\right) ; \psi\right)\right), \\
\not \partial \psi=\mathcal{A}\left(d \phi\left(e_{\alpha}\right), e_{\alpha} \cdot \psi\right),
\end{gathered}
$$

where $\not \partial$ is the usual Dirac operator, $A$ is the second fundamental form of $N$ in $\mathbb{R}^{K}$, and

$$
\begin{aligned}
\mathcal{A}\left(d \phi\left(e_{\alpha}\right), e_{\alpha} \cdot \psi\right) & :=\left(\nabla \phi^{i} \cdot \psi^{j}\right) \otimes A\left(\partial_{y^{i}}, \partial_{y^{j}}\right), \\
\operatorname{Re}\left(P\left(\mathcal{A}\left(d \phi\left(e_{\alpha}\right), e_{\alpha} \cdot \psi\right) ; \psi\right)\right) & :=P\left(A\left(\partial_{y^{l}}, \partial_{y^{j}}\right) ; \partial_{y^{i}}\right) \operatorname{Re}\left(\left\langle\psi^{i}, d \phi^{l} \cdot \psi^{j}\right\rangle\right) .
\end{aligned}
$$

Here $P(\xi ; \cdot)$ denotes the shape operator, defined by $\langle P(\xi ; X), Y\rangle=\langle A(X, Y), \xi\rangle$ for $X, Y \in$ $\Gamma(T N)$, and $\operatorname{Re}(z)$ denotes the real part of $z \in \mathbb{C}$. We refer to $[2,3,5,11,24,30,33]$ for more details.

Denote

$$
W^{2,2}(M, N):=\left\{\phi \in W^{2,2}\left(M, \mathbb{R}^{K}\right) \text { with } \phi(x) \in N \text { for a.e. } x \in M\right\},
$$

$W^{1,4 / 3}\left(M, \Sigma M \otimes \phi^{\star} T N\right):=\left\{\psi \in W^{1,4 / 3}\left(M, \Sigma M \otimes \mathbb{R}^{K}\right)\right.$ with $\psi(x) \in \Sigma M \otimes \phi^{\star} T N$ for a.e. $x \in M\}$.

In this paper, with applications for the Dirac-harmonic heat flow in mind, we want to consider pairs $(\phi, \psi)$ that satisfy the Euler-Lagrange equations up to an error term in $L^{1}$. Here is the precise

Definition 1.1 $(\phi, \psi) \in W^{2,2}(M, N) \times W^{1, \frac{4}{3}}\left(M, \Sigma M \times \phi^{\star} T N\right)$ is called an approximate Dirac-harmonic map if there exist $(\tau(\phi, \psi), h(\phi, \psi)) \in L^{1}(M)$ such that

$$
\begin{gathered}
\tau(\phi, \psi)=\Delta \phi-A(d \phi, d \phi)-\operatorname{Re}\left(P\left(\mathcal{A}\left(d \phi\left(e_{\alpha}\right), e_{\alpha} \cdot \psi\right) ; \psi\right)\right), \\
h(\phi, \psi)=\not \partial \psi \mathcal{A}\left(d \phi\left(e_{\alpha}\right), e_{\alpha} \cdot \psi\right) .
\end{gathered}
$$


Thus, $(\phi, \psi)$ is a Dirac-harmonic map if and only if $\tau(\phi, \psi)=h(\phi, \psi)=0$. In the sequel, we shall need to assume that the error terms are in stronger spaces than $L^{1}$, however. See for instance Theorem 1.2.

As for harmonic maps, the conformal invariance of the energy functional $L$ leads to non-compactness of the set of Dirac-harmonic maps in dimension 2. This has been studied extensively by $[2,18,32]$, and in [11] for a more general case. For the harmonic map case, we refer to [7,15-17,22,29,31]. Roughly speaking, the results of those papers assert that the failure of strong convergence occurs at finitely many concentration points of the energy. At such points, finitely many bubbles (i.e. nonconstant Dirac-harmonic spheres) separate, and the total energies from these bubbles account for the total loss of Dirichlet energies during the process of convergence. Moreover, the image of the remaining the base map and those of the bubbles are connected in the target manifold. This is called the no neck property.

In this paper, we will extend the results from $[2,18,32]$ to the approximate Dirac-harmonic maps from a closed Riemann surface $M$ to a compact Riemannian manifold $N$.

Denote the energy of $\phi$ on $\Omega \subset M$ by

$$
E(\phi ; \Omega)=\frac{1}{2} \int_{\Omega}|\nabla \phi|^{2} d M,
$$

the energy of $\psi$ on $\Omega \subset M$ by

$$
E(\psi ; \Omega)=\int_{\Omega}|\psi|^{4} d M
$$

and the energy of the pair $(\phi, \psi)$ on $\Omega \subset M$ by

$$
E(\phi, \psi ; \Omega)=\int_{\Omega}\left(|\nabla \phi|^{2}+|\psi|^{4}\right) d M .
$$

We shall often omit the domain $M$ from the notation and simply write $E(\phi)=$ $E(\phi ; M), E(\psi)=E(\psi ; M)$ and $E(\phi, \psi)=E(\phi, \psi ; M)$.

Our first main result is

Theorem 1.2 For a sequence of smooth approximate Dirac-harmonic maps $\left\{\left(\phi_{n}, \psi_{n}\right)\right\}$ from a closed Riemann surface $M$ to a compact Riemannian manifold $N$ with uniformly bounded energy

$$
E\left(\phi_{n}, \psi_{n}\right) \leq \Lambda<\infty
$$

and

$$
\left\|\tau\left(\phi_{n}, \psi_{n}\right)\right\|_{L^{2}}+\left\|h\left(\phi_{n}, \psi_{n}\right)\right\|_{L^{4}} \leq \Lambda,
$$

defining the blow-up set

$$
\mathcal{S}:=\cap_{r>0}\left\{x \in M \mid \liminf _{n \rightarrow \infty} \int_{D(x, r)}\left(\left|d \phi_{n}\right|^{2}+\left|\psi_{n}\right|^{4}\right) \geq \frac{\epsilon_{0}^{2}}{2}\right\},
$$

where $\epsilon_{0}>0$ is as in Theorem 2.1, then $\mathcal{S}$ is a finite set $\left\{p_{1}, \ldots, p_{I}\right\}$. There exists an approximate Dirac-harmonic map $(\phi, \psi)$ so that, up to a subsequence, still denoted by $\left\{\left(\phi_{n}, \psi_{n}\right)\right\}$, converges weakly in $W_{l o c}^{2,2}(M \backslash \mathcal{S}) \times W_{l o c}^{1,2}(M \backslash \mathcal{S})$ to $(\phi, \psi)$ and there are a finite set of Dirac-harmonic spheres $\left(\sigma_{i}^{l}, \xi_{i}^{l}\right): S^{2} \rightarrow N, i=1, \ldots, I ; l=1, \ldots, L_{i}$ such that

$$
\lim _{n \rightarrow \infty} E\left(\phi_{n}\right)=E(\phi)+\sum_{i=1}^{I} \sum_{l=1}^{L_{i}} E\left(\sigma_{i}^{l}\right)
$$




$$
\lim _{n \rightarrow \infty} E\left(\psi_{n}\right)=E(\psi)+\sum_{i=1}^{I} \sum_{l=1}^{L_{i}} E\left(\xi_{i}^{l}\right)
$$

and the image $\phi(M) \cup_{i=1}^{I} \cup_{l=1}^{L_{i}}\left(\sigma_{i}^{l}\left(S^{2}\right)\right)$ is a connected set.

Remark 1.3 From the proof of Theorem 4.1 in Sect. 4, it is easy to see that also the following identities hold:

$$
\begin{gathered}
\lim _{n \rightarrow \infty} \int_{M}\left|\nabla \psi_{n}\right|^{\frac{4}{3}} d M=\int_{M}|\nabla \psi|^{\frac{4}{3}} d M+\sum_{i=1}^{I} \sum_{l=1}^{L_{i}} \int_{S^{2}}\left|\nabla \xi_{l}^{i}\right|^{\frac{4}{3}} d S^{2}, \\
\lim _{n \rightarrow \infty} L\left(\phi_{n}, \psi_{n}\right)=L(\phi, \psi)+\sum_{i=1}^{I} \sum_{l=1}^{L_{i}} L\left(\sigma_{i}^{l}, \xi_{i}^{l}\right) .
\end{gathered}
$$

This is due to the fact that both $\int_{M}|\nabla \psi|^{\frac{4}{3}} d M$ and $L(\phi, \psi)$ are conformally invariant [3].

As an application of Theorem 1.2, we study the asymptotic behavior at infinite time for the Dirac-harmonic map flow in dimension 2.

For that purpose, we first review the heat flow for Dirac-harmonic maps as introduced and studied in [4,12] (a different flow has been introduced and studied in [1]). One tries to find $(\phi, \psi): M \times[0, \infty) \rightarrow N \times \phi^{\star} T N$ that solves

$$
\begin{cases}\partial_{t} \phi=\tau(\phi)-\operatorname{Re}\left(P\left(\mathcal{A}\left(d \phi\left(e_{\alpha}\right), e_{\alpha} \cdot \psi\right) ; \psi\right),\right. & \text { in } M \times(0, \infty) ; \\ \partial \psi=\mathcal{A}\left(d \phi\left(e_{\alpha}\right), e_{\alpha} \cdot \psi\right), & \text { in } M \times(0, \infty) .\end{cases}
$$

with the following boundary-initial data:

$$
\begin{cases}\phi(x, t)=\varphi(x), & \text { on } \partial M \times[0, \infty) ; \\ \phi(x, 0)=\phi_{0}(x), & \text { in } M ; \\ \mathcal{B} \psi(x, t)=\mathcal{B} \chi(x), & \text { on } \partial M \times[0, \infty) ; \\ \phi_{0}(x)=\varphi(x), & \text { on } \partial M,\end{cases}
$$

where $\tau(\phi)=\Delta \phi-A(\phi)(d \phi, d \phi)$ is the tension field of $\phi, M$ is a compact Riemannian spin manifold with smooth boundary $\partial M, \phi_{0} \in W^{1,2}(M, N), \varphi \in C^{2+\alpha}(\partial M ; N), \chi \in$ $C^{1+\alpha}\left(\partial M ; \Sigma M \otimes \phi^{\star} T N\right)$ are given maps and $\mathcal{B}=\mathcal{B}^{ \pm}$is the chiral boundary operator defined as follows:

$$
\begin{aligned}
& \mathcal{B}^{ \pm}: L^{2}\left(M,\left.\Sigma M \otimes \phi^{\star} T N\right|_{\partial M}\right) \rightarrow L^{2}\left(M,\left.\Sigma M \otimes \phi^{\star} T N\right|_{\partial M}\right) \\
& \psi \mapsto \frac{1}{2}(I d \pm \vec{n} \cdot G) \cdot \psi,
\end{aligned}
$$

where $\vec{n}$ is the outward unit normal vector field on $\partial M$, and $G$ is the chiral operator satisfying:

$$
G^{2}=I d, \quad G^{*}=G, \quad \nabla G=0, \quad G X \cdot=-X \cdot G,
$$

for any $X \in \Gamma(T M)$.

In dimension $\operatorname{dim} M=2$, [4] established the short-time existence for (1.14) with smooth initial-boundary data (1.15). Later, [12] showed that, under some smallness assumption for $\left\|\phi_{0}\right\|_{H^{1}}+\|\mathcal{B} \chi\|_{L^{2}}$, there exists a unique global weak solution to (1.14) with initial-boundary data (1.15), which has at most finitely many singular times and enjoys the property 


$$
\begin{aligned}
& E(\phi(t), \psi(t) ; M)+\int_{0}^{t} \int_{M \times\{s\}}\left|\partial_{t} \phi\right|^{2} d M d s \\
& \quad \leq C\left(M, E\left(\phi_{0}\right),\left\|\mathcal{B} \psi_{0}\right\|_{L^{2}(\partial M)}\right), \quad \forall 0 \leq t<\infty .
\end{aligned}
$$

It follows from (1.19) that there exists a sequence $t_{n} \uparrow \infty$ such that $\left(\phi_{n}, \psi_{n}\right):=$ $\left(\phi\left(\cdot, t_{n}\right), \psi\left(\cdot, t_{n}\right)\right) \in W^{2,2}(M, N) \times W^{1, \frac{4}{3}}\left(M, \Sigma M \times \phi^{\star} T N\right)$ is an approximate Diracharmonic map with boundary-data

$$
\left\{\begin{array}{lll}
\phi(x)=\varphi(x), & \text { on } & \partial M \\
\mathcal{B} \psi(x)=\mathcal{B} \chi(x), & \text { on } & \partial M,
\end{array}\right.
$$

which satisfies the assumptions of Theorem 1.2. In fact, $h\left(\phi_{n}, \psi_{n}\right)=0$ and

$$
\tau\left(\phi_{n}, \psi_{n}\right):=\partial_{t} \phi\left(\cdot, t_{n}\right) \text { satisfying }\left\|\tau\left(\phi_{n}, \psi_{n}\right)\right\|_{L^{2}} \rightarrow 0 .
$$

Thus, as an immediate corollary, we obtain

Theorem 1.4 For $\operatorname{dim} M=2$ and $\phi_{0} \in H^{1}(M, N), \varphi \in C^{2+\alpha}(\partial M, N), \chi \in$ $C^{1+\alpha}\left(\partial M, \Sigma M \otimes \varphi^{\star} T N\right)$, let $(\phi, \psi): M \times[0, \infty) \rightarrow N \times \phi^{\star} T N$ be a global weak solution of (1.14) and (1.15), which has finitely many singular times and satisfies (1.19). Moreover, we assume that $(\phi, \psi)$ blows up at infinite time and at interior points. Then there exist $t_{n} \uparrow \infty$, a Dirac-harmonic map $\left(\phi_{\infty}, \psi_{\infty}\right) \in C^{2+\alpha}(M, N) \times C^{1+\alpha}\left(M, \Sigma M \otimes \phi_{\infty}^{*} T N\right)$ with boundary data $\left.\phi_{\infty}\right|_{\partial M}=\varphi$ and $\left.\mathcal{B} \psi_{\infty}\right|_{\partial M}=\mathcal{B} \chi$, and a nonnegative integer I and finitely many points $\left\{p_{1}, \ldots, p_{I}\right\} \in M$ such that

$(1)\left(\phi_{n}, \psi_{n}\right):=\left(\phi\left(\cdot, t_{n}\right), \psi\left(\cdot, t_{n}\right)\right) \rightarrow\left(\phi_{\infty}, \psi_{\infty}\right)$ in $W^{1,2}(M, N) \times W^{1, \frac{4}{3}}(M, \Sigma M \times$ $\left.\phi^{\star} T N\right)$;

(2) $\left(\phi_{n}, \psi_{n}\right) \rightarrow\left(\phi_{\infty}, \psi_{\infty}\right)$ in $W_{l o c}^{1,2}\left(M \backslash\left\{p_{1}, \ldots, p_{L}\right\}\right) \times L_{l o c}^{4}\left(M \backslash\left\{p_{1}, \ldots, p_{I}\right\}\right)$;

(3) For $1 \leq i \leq I$, there exist a positive integer $L_{i}$ and $L_{i}$ nontrivial Dirac-harmonic spheres $\left(\sigma_{i}^{l}, \xi_{i}^{l}\right): S^{2} \rightarrow N, i=1, \ldots, I ; l=1, \ldots, L_{i}$ such that

$$
\begin{aligned}
& \lim _{n \rightarrow \infty} E\left(\phi_{n}\right)=E\left(\phi_{\infty}\right)+\sum_{i=1}^{I} \sum_{l=1}^{L_{i}} E\left(\sigma_{i}^{l}\right), \\
& \lim _{n \rightarrow \infty} E\left(\psi_{n}\right)=E\left(\psi_{\infty}\right)+\sum_{i=1}^{I} \sum_{l=1}^{L_{i}} E\left(\xi_{i}^{l}\right),
\end{aligned}
$$

and

$$
\lim _{n \rightarrow \infty}\left\|\phi\left(\cdot, t_{n}\right)-\phi_{\infty}(\cdot)-\sigma_{i}^{l}(\cdot)\right\|_{L^{\infty}(M)}=0 .
$$

Remark 1.5 In this Theorem 1.4, we only consider the interior blow-up phenomenon for the Dirac-harmonic maps flow. The boundary blow-up case is treated in a subsequent paper [13].

This paper is organized as follows. In Sect. 2, we shall prove some basic lemmas, called small energy regularity, Pohozaev's identity and removable singularity, so that the expert will readily know what we are talking about, and we shall recall some known results for later use. In Sect. 3, we shall establish the three circle theorem for approximate Dirac-harmonic maps which ensures the exponential decay of the tangential energy. Our main result Theorem 1.2 will be proved in Sect. 4. 


\section{Some basic lemmas}

In this section, we will prove some basic lemmas and recall some known results which will be used in this paper.

Firstly, we prove a small energy regularity theorem.

Theorem 2.1 There is a small constant $\epsilon_{0}>0$ such that if $(\phi, \psi) \in W^{2, p}(D, N) \times$ $W^{1, q}\left(D, \Sigma D \otimes \phi^{\star} T N\right)$ is an approximate Dirac-harmonic map from the unit disc $D$ in $\mathbb{R}^{2}$ to a compact Riemannian manifold $(N, g)$ with $\tau \in L^{p}, h \in L^{q}$ for some $\frac{4}{3} \leq p \leq 2, \frac{8}{5} \leq q \leq 2$, and satisfies

$$
E(\phi, \psi ; D)=\int_{D}\left(|d \phi|^{2}+|\psi|^{4}\right) d x<\epsilon_{0}^{2},
$$

then

$$
\begin{aligned}
\|\phi-\bar{\phi}\|_{W^{2, p}\left(D_{\frac{1}{2}}\right)} & \leq C\left(\|d \phi\|_{L^{2}(D)}+\|\tau\|_{L^{p}(D)}\right), \\
\|\psi\|_{W^{1, q}\left(D_{\frac{1}{2}}\right)} & \leq C\left(\|\psi\|_{L^{4}(D)}+\|h\|_{L^{q}(D)}\right),
\end{aligned}
$$

where $\bar{\phi}:=\frac{1}{\left|D_{1 / 2}\right|} \int_{D_{1 / 2}} \phi d x$ and $C>0$ is a constant depending only on $p, q, \Lambda, N$.

Moreover, by the Sobolev embedding $W^{2, p}\left(\mathbb{R}^{2}\right) \subset C^{0}\left(\mathbb{R}^{2}\right)$, we have

$$
\begin{aligned}
\|\phi\|_{O s c\left(D_{1 / 2}\right)} & =\sup _{x, y \in D_{1 / 2}}|\phi(x)-\phi(y)| \\
& \leq C(\Lambda, N)\left(\|\nabla \phi\|_{L^{2}(D)}+\|\tau(u)\|_{L^{p}(D)}\right) .
\end{aligned}
$$

Proof Without loss of generality, we assume $\frac{1}{\left|D_{1 / 2}\right|} \int_{D_{1 / 2}} \phi d x=0$.

Choosing a cut-off function $\eta \in C_{0}^{\infty}(D)$ satisfying $0 \leq \eta \leq 1,\left.\eta\right|_{D_{3 / 4}} \equiv 1,|\nabla \eta|+$ $\left|\nabla^{2} \eta\right| \leq C$, by the standard theory of first order elliptic equations, for any $1<q<2$, we have

$$
\begin{aligned}
\|\eta \psi\|_{W^{1, q}(D)} & \leq C\|\not \partial(\eta \psi)\|_{L^{q}(D)} \\
& \leq C\|\nabla \eta \cdot \psi+\eta \not \gamma\|_{L^{q}(D)} \\
& \leq C\left(\|\psi\|_{L^{q}(D)}+\left\|\left|d \phi\|\eta \psi \mid\|_{L^{q}(D)}+\|h\|_{L^{q}(D)}\right)\right.\right. \\
& \leq C\|d \phi\|_{L^{2}(D)}\|\eta \psi\|_{L^{\frac{2 q}{2-q}(D)}}+C\left(\|\psi\|_{L^{q}(D)}+\|h\|_{L^{q}(D)}\right) \\
& \leq C \epsilon_{0}\|\eta \psi\|_{L^{\frac{2 q}{2-q}}(D)}+C\left(\|\psi\|_{L^{q}(D)}+\|h\|_{L^{q}(D)}\right) .
\end{aligned}
$$

Taking $q=\frac{8}{5}$ and $\epsilon_{0}>0$ sufficiently small, by Sobolev embedding, we obtain

$$
\|\eta \psi\|_{L^{8}(D)} \leq C\|\eta \psi\|_{W^{1,8 / 5}(D)} \leq C\left(\|\psi\|_{L^{q}(D)}+\|h\|_{L^{q}(D)}\right) .
$$

Computing directly, we get

$$
\begin{aligned}
|\Delta(\eta \phi)| & =|\eta \Delta \phi+2 \nabla \eta \nabla \phi+\phi \Delta \eta| \\
& \leq C\left(|\phi|+|d \phi|+|d \phi||\eta d \phi|+|\psi|^{2}|\eta d \phi|+|\tau|\right) \\
& \leq C|d \phi||d(\eta \phi)|+C\left(|\phi|+|d \phi|+\eta|\psi|^{2}|d \phi|+|\tau|\right) .
\end{aligned}
$$


By standard elliptic estimates,

$$
\begin{aligned}
\|\eta \phi\|_{W^{2,4 / 3}(D) \leq} & C\left\|\left|d \phi\|d(\eta \phi) \mid\|_{L^{4 / 3}(D)}\right.\right. \\
& +C\left(\|d \phi\|_{L^{4 / 3}(D)}+\left\||\eta \psi|^{2}|d \phi|\right\|_{L^{4 / 3}(D)}+\||\tau|\|_{L^{4 / 3}(D)}\right) \\
\leq & C\|d(\eta \phi)\|_{L^{4}(D)}\|d \phi\|_{L^{2}(D)} \\
& +C\left(\|d \phi\|_{L^{4 / 3}(D)}+\|\eta \psi\|_{L^{8}(D)}^{2}\|d \phi\|_{L^{2}(D)}+\||\tau|\|_{L^{4 / 3}(D)}\right) \\
\leq & C \epsilon_{0}\|d(\eta \phi)\|_{L^{4}(D)}+C\left(\|d \phi\|_{L^{2}(D)}+\||\tau|\|_{L^{4 / 3}(D)}\right) .
\end{aligned}
$$

Taking $\epsilon_{0}>0$ sufficiently small, we have

$$
\|d \phi\|_{L^{4}\left(D_{3 / 4}\right)} \leq C\|\eta \phi\|_{W^{2,4 / 3}(D)} \leq C\left(\|d \phi\|_{L^{2}(D)}+\|\tau\|_{L^{4 / 3}(D)}\right) .
$$

By the interior elliptic estimates, for any $\frac{4}{3} \leq p \leq 2$ we have

$$
\begin{aligned}
\|\phi\|_{W^{2, p}\left(D_{1 / 2}\right)} \leq & C\left(\|\Delta \phi\|_{L^{p}\left(D_{3 / 4}\right)}+\|d \phi\|_{L^{p}\left(D_{3 / 4}\right)}\right) \\
\leq & C\left(\|d \phi\|_{L^{4}\left(D_{3 / 4}\right)}^{2}+\|\psi\|_{L^{8}\left(D_{3 / 4}\right)}^{2}\|d \phi\|_{L^{4}\left(D_{3 / 4}\right)}\right. \\
& \left.+\||\tau|\|_{L^{p}(D)}+\|d \phi\|_{L^{p}\left(D_{3 / 4}\right)}\right) \\
\leq & C\left(\|d \phi\|_{L^{2}(D)}+\|\tau\|_{L^{p}(D)}\right) .
\end{aligned}
$$

By the interior elliptic estimates for first order equations, for any $\frac{8}{5} \leq q \leq 2$, we get

$$
\begin{aligned}
\|\psi\|_{W^{1, q}\left(D_{1 / 2}\right)} & \leq C\left(\|\not \psi\|_{L^{q}\left(D_{3 / 4}\right)}+\|\psi\|_{L^{q}\left(D_{3 / 4}\right)}\right) \\
& \leq C\left(\|d \phi\|_{L^{4}\left(D_{3 / 4}\right)}\|\psi\|_{L^{4}\left(D_{3 / 4}\right)}+\|\psi\|_{L^{2}(D)}+\|h\|_{L^{q}(D)}\right) \\
& \leq C\left(1+\|d \phi\|_{L^{2}(D)}+\|\tau\|_{L^{p}(D)}\right)\left(\|\psi\|_{L^{4}(D)}+\|h\|_{L^{q}(D)}\right) \\
& \leq C\left(\|\psi\|_{L^{4}(D)}+\|h\|_{L^{q}(D)}\right) .
\end{aligned}
$$

Secondly, by a direct computation, we have the following proposition.

Proposition 2.2 Suppose $\phi \in C^{2}(M, N), \psi \in C^{2}\left(M, \Sigma M \otimes \phi^{\star} T N\right)$. Let $\left\{e_{\alpha}\right\}_{\alpha=1}^{2}$ be a unit normal basis of $T M$ and $e_{\beta} \in \Gamma(T M)$ a section satisfying

$$
\left[e_{\beta}, e_{\alpha}\right]=0, \alpha=1,2 \text {, }
$$

then

$$
\begin{aligned}
\left\langle\psi, \widetilde{\nabla}_{e_{\beta}}(\not D \psi)\right\rangle= & 2\left\langle\operatorname{Re}\left(P\left(\mathcal{A}\left(d \phi\left(e_{\alpha}\right), e_{\alpha} \cdot \psi\right) ; \psi\right)\right),\right. \\
& \left.\phi_{\beta}\right\rangle+\left\langle\psi, \not D \psi_{\beta}\right\rangle,
\end{aligned}
$$

where [·, ·] is the Lie bracket, $\phi_{\beta}=d \phi\left(e_{\beta}\right)$ and $\psi_{\beta}=\widetilde{\nabla}_{e_{\beta}} \psi$.

Proof Proposition 2.2 is proved in [3]. For the reader's convenience, we recall it here.

On the one hand, we have

$$
\begin{aligned}
\left\langle\operatorname{Re}\left(P\left(\mathcal{A}\left(d \phi\left(e_{\alpha}\right), e_{\alpha} \cdot \psi\right) ; \psi\right)\right), \phi_{\beta}\right\rangle & \left.=\left\langle\frac{1}{2} R_{l i j}^{m}(\phi)\left\langle\psi^{i}, \nabla \phi^{l} \cdot \psi^{j}\right\rangle \partial_{y^{m}}, \phi_{\beta}^{p} \partial_{y}\right\rangle\right\rangle \\
& =\frac{1}{2} R_{m l i j}\left\langle\psi^{i}, \nabla \phi^{l} \cdot \psi^{j}\right\rangle \phi_{\beta}^{m} .
\end{aligned}
$$


On the other hand, by a direct computation, we get

$$
\widetilde{\nabla}_{e_{\alpha}} \widetilde{\nabla}_{e_{\beta}} \psi-\widetilde{\nabla}_{e_{\beta}} \widetilde{\nabla}_{e_{\alpha}} \psi=R^{\Sigma M}\left(e_{\alpha}, e_{\beta}\right) \psi^{i} \otimes \partial y^{i}+R_{l i j}^{m} \phi_{\alpha}^{i} \phi_{\beta}^{j} \psi^{l} \otimes \partial y^{m}
$$

where $R^{\Sigma M}$ is the curvature operator of the connection on the spinor bundle $\Sigma M$. For this curvature, we have (see $[3,6,14]$ )

$$
e_{\alpha} \cdot R^{\Sigma M}\left(e_{\alpha}, X\right) \psi^{i}=\frac{1}{2} \operatorname{Ric}(X), \forall X \in \Gamma(M) .
$$

Thus we obtain

$$
\begin{aligned}
\left\langle\psi, e_{\alpha} \cdot R^{\Sigma M}\left(e_{\alpha}, e_{\beta}\right) \psi^{i} \otimes \partial y^{i}\right\rangle & =g_{i j}\left\langle\psi^{j}, e_{\alpha} \cdot R^{\Sigma M}\left(e_{\alpha}, e_{\beta}\right) \psi^{i}\right\rangle \\
& =\frac{1}{2} g_{i j}\left\langle\psi^{j}, \operatorname{Ric}\left(e_{\beta}\right) \cdot \psi^{i}\right\rangle=0 .
\end{aligned}
$$

Therefore,

$$
\begin{aligned}
\left\langle\psi, \not D \psi_{\beta}\right\rangle & =\left\langle\psi, e_{\alpha} \cdot \widetilde{\nabla}_{e_{\alpha}} \widetilde{\nabla}_{e_{\beta}} \psi\right\rangle \\
& =\left\langle\psi, \widetilde{\nabla}_{e_{\beta}}\left(e_{\alpha} \cdot \widetilde{\nabla}_{e_{\alpha}} \psi\right)\right\rangle+R_{l i j}^{m} \phi_{\beta}^{j}\left\langle\psi, \nabla \phi^{i} \cdot \psi^{l} \otimes \partial_{y^{m}}\right\rangle \\
& =\left\langle\psi, \widetilde{\nabla}_{e_{\beta}}(\not D \psi)\right\rangle+R_{m l i j} \phi_{\beta}^{j}\left\langle\psi^{m}, \nabla \phi^{i} \cdot \psi^{l}\right\rangle \\
& =\left\langle\psi, \widetilde{\nabla}_{e_{\beta}}(\not D \psi)\right\rangle-R_{m l i j}\left\langle\psi^{i}, \nabla \phi^{l} \cdot \psi^{j}\right\rangle \phi_{\beta}^{m} .
\end{aligned}
$$

Then, the conclusion of the proposition follows immediately.

Thirdly, we compute Pohozaev's identity for approximate Dirac-harmonic maps.

Lemma 2.3 Let $D \subset \mathbb{R}^{2}$ be the unit disk and $(\phi, \psi)$ be a smooth approximate Diracharmonic map, then for any $0<t<\frac{1}{2}$, we have

$$
\begin{aligned}
t \int_{\partial D_{t}}\left(\left|\phi_{r}\right|^{2}-\frac{1}{2}|\nabla \phi|^{2}\right)= & \frac{1}{2} \int_{\partial D_{t}}\left\langle\psi, r^{-1} \frac{\partial}{\partial \theta} \cdot \psi_{\theta}\right\rangle-\frac{1}{2} \int_{D_{t}}\langle\psi, \not D \psi\rangle d x \\
& -\operatorname{Re} \int_{D_{t}}\left\langle\not D \psi, r \psi_{r}\right\rangle d x+\int_{D_{t}} r \phi_{r} \tau d x,
\end{aligned}
$$

where $(r, \theta)$ are polar coordinates in $D$ centered at $0, \phi_{r}=d \phi\left(\frac{\partial}{\partial r}\right), \psi_{r}=\widetilde{\nabla}_{\frac{\partial}{\partial r}} \psi$ and $\psi_{\theta}=\widetilde{\nabla}_{\frac{\partial}{\partial \theta}} \psi$.

Proof Multiplying the equation (1.7) by $r \phi_{r}$ and integrating over $D_{t}$, by Proposition 2.2 and the fact that $r \phi_{r}=x^{\beta} \frac{\partial \phi}{\partial x^{\beta}}=x^{\beta} d \phi\left(\frac{\partial}{\partial x^{\beta}}\right)$, we get

$$
\begin{aligned}
\int_{D_{t}} r \phi_{r} \tau d x & =\int_{D_{t}} r \phi_{r} \Delta \phi d x-\int_{D_{t}}\left\langle r \phi_{r}, \operatorname{Re}\left(P\left(\mathcal{A}\left(d \phi\left(e_{\alpha}\right), e_{\alpha} \cdot \psi\right) ; \psi\right)\right)\right\rangle d x \\
& =\int_{D_{t}} r \phi_{r} \Delta \phi d x+\frac{1}{2} \int_{D_{t}}\left\langle x^{\beta} \psi, \not D \psi_{\beta}\right\rangle d x-\frac{1}{2} \int_{D_{t}}\left\langle x^{\beta} \psi, \widetilde{\nabla} \frac{\partial}{\partial x^{\beta}} \not D \psi\right\rangle d x \\
: & =\mathbb{I}+\mathbb{I I}+\mathbb{I I I I} .
\end{aligned}
$$


On the one hand, integrating by parts, we have

$$
\begin{aligned}
\mathbb{I} & =\int_{\partial D_{t}} r\left|\phi_{r}\right|^{2}-\int_{D_{t}} \nabla \phi \nabla\left(r \phi_{r}\right) d x \\
& =\int_{\partial D_{t}} r\left|\phi_{r}\right|^{2}-\int_{D_{t}}|\nabla \phi|^{2} d x-\frac{1}{2} \int_{D_{t}} r \frac{\partial}{\partial r}|\nabla \phi|^{2} d x \\
& =r \int_{\partial D_{t}}\left(\left|\phi_{r}\right|^{2}-\frac{1}{2}|\nabla \phi|^{2}\right) .
\end{aligned}
$$

On the other hand, by Lemma 2.6, we get

$$
\begin{aligned}
2 \mathbb{I I} & =\int_{D_{t}}\left\langle x^{\beta} \psi, \not D \psi_{\beta}\right\rangle d x \\
& =\int_{D_{t}}\left\langle\not D\left(x^{\beta} \psi\right), \psi_{\beta}\right\rangle d x-\int_{\partial D_{t}}\left\langle\vec{n} \cdot x^{\beta} \psi, \psi_{\beta}\right\rangle d x \\
& =-\int_{D_{t}}\langle\psi, \not D \psi\rangle d x+\int_{D_{t}}\left\langle\not D \psi, r \psi_{r}\right\rangle d x+\int_{\partial D_{t}}\left\langle\psi, r \frac{\partial}{\partial r} \cdot \psi_{r}\right\rangle,
\end{aligned}
$$

and

$$
\begin{aligned}
2 \mathbb{I I I I} & =-\int_{D_{t}}\left\langle x^{\beta} \psi, \widetilde{\nabla}_{\partial_{\beta}} \not D \psi\right\rangle d x \\
& =-\int_{\partial D_{t}}\langle r \psi, \not D \psi\rangle d x+\int_{D_{t}}\left\langle\widetilde{\nabla}_{\partial_{\beta}}\left(x^{\beta} \psi\right), \not D \psi\right\rangle d x \\
& =2 \int_{D_{t}}\langle\psi, \not D \psi\rangle d x+\int_{D_{t}}\left\langle r \psi_{r}, \not D \psi\right\rangle d x-\int_{\partial D_{t}}\langle r \psi, \not D \psi\rangle .
\end{aligned}
$$

Combining the preceding equations, we get the conclusion of the lemma.

Corollary 2.4 Under the assumption of Lemma 2.3, if $\|\tau(\phi, \psi)\|_{L^{2}(D)}+\|h(\phi, \psi)\|_{L^{4}(D)} \leq$ $C$, then for any $0<t<\frac{1}{4}$, we have

$$
\begin{aligned}
& \int_{D_{2 t} \backslash D_{t}}\left(\left|\phi_{r}\right|^{2}-\frac{1}{2}|\nabla \phi|^{2}\right) d x \\
& \quad \leq C\left(\left\|r^{-1} \frac{\partial \psi}{\partial \theta}\right\|_{L^{\frac{4}{3}}\left(D_{2 t} \backslash D_{t}\right)}+\left\|r^{-1} \frac{\partial \phi}{\partial \theta}\right\|_{L^{2}\left(D_{2 t} \backslash D_{t}\right)}\right)+C t .
\end{aligned}
$$

Proof By Lemma 2.3, for any $0<s<\frac{1}{2}$, we have

$$
s \int_{\partial D_{s}}\left(\left|\phi_{r}\right|^{2}-\frac{1}{2}|\nabla \phi|^{2}\right):=\mathbb{J}_{1}+\cdots+\mathbb{J}_{4} .
$$

It is easy to see that

$$
\begin{aligned}
& \mathbb{J}_{2}=-\frac{1}{2} \int_{D_{s}}\langle\psi, \not D \psi\rangle d x \leq C s\|\psi\|_{L^{4}\left(D_{s}\right)}\|h\|_{L^{4}\left(D_{s}\right)} \leq C s, \\
& \mathbb{J}_{4}=\int_{D_{s}} r \phi_{r} \tau d x \leq C s\|d \phi\|_{L^{2}\left(D_{s}\right)}\|\tau\|_{L^{2}\left(D_{s}\right)} \leq C s,
\end{aligned}
$$


and

$$
\begin{aligned}
\mathbb{J}_{3} & =-\operatorname{Re} \int_{D_{s}}\left\langle\not D \psi, r \psi_{r}\right\rangle d x \\
& \leq C s\left\|\psi_{r}\right\|_{L^{\frac{4}{3}\left(D_{s}\right)}}\|h\|_{L^{4}\left(D_{s}\right)} \\
& \leq C s\left(\|\nabla \psi\|_{L^{\frac{4}{3}\left(D_{s}\right)}}+\|\psi\|_{L^{4}\left(D_{s}\right)}\|d \phi\|_{L^{2}\left(D_{s}\right)}\right)\|h\|_{L^{4}\left(D_{s}\right)} \\
& \leq C s
\end{aligned}
$$

where we used the fact that

$$
\begin{aligned}
& \left|\psi_{r}\right| \leq C\left(\left|\frac{\partial \psi}{\partial r}\right|+|\psi|\left|\frac{\partial \phi}{\partial r}\right|\right), \\
& \left|\psi_{\theta}\right| \leq C\left(\left|\frac{\partial \psi}{\partial \theta}\right|+|\psi|\left|\frac{\partial \phi}{\partial \theta}\right|\right) .
\end{aligned}
$$

Multiplying (2.7) by $\frac{1}{s}$ and integrating from $t$ to $2 t$, we get

$$
\begin{aligned}
\int_{D_{2 t} \backslash D_{t}}\left(\left|\phi_{r}\right|^{2}-\frac{1}{2}|\nabla \phi|^{2}\right) & \leq \int_{t}^{2 t} \frac{1}{2 s} \int_{\partial D_{s}}\left\langle\psi, r^{-1} \frac{\partial}{\partial \theta} \cdot \psi_{\theta}\right\rangle d \theta d s+C t \\
& \leq C\left\|r^{-1} \psi_{\theta}\right\|_{L^{\frac{4}{3}}\left(D_{2 t} \backslash D_{t}\right)}\|\psi\|_{L^{4}\left(D_{2 t} \backslash D_{t}\right)}+C t \\
& \leq C\left(\left\|r^{-1} \frac{\partial \psi}{\partial \theta}\right\|_{L^{\frac{4}{3}\left(D_{2 t} \backslash D_{t}\right)}}+\left\|r^{-1} \frac{\partial \phi}{\partial \theta}\right\|_{L^{2}\left(D_{2 t} \backslash D_{t}\right)}\right)+C t .
\end{aligned}
$$

Thirdly, we state an interior removable singularity result.

Theorem 2.5 Let $(\phi, \psi) \in W_{l o c}^{2,2}(D \backslash\{0\}) \times W_{l o c}^{1,2}(D \backslash\{0\})$ be an approximate Diracharmonic map from $D \backslash\{0\}$ to $N$ with finite energy

$$
\|d \phi\|_{L^{2}(D)}+\|\psi\|_{L^{4}(D)} \leq C
$$

that satisfies

$$
\begin{array}{ll}
\tau=f \in L^{2}(D), & x \in D \backslash\{0\}, \\
h=g \in L^{2}(D), & x \in D \backslash\{0\},
\end{array}
$$

then $(\phi, \psi)$ can be extended to a field in $W^{2,2}(D) \times W^{1,2}(D)$.

Proof By a standard argument as in Lemma A.2 in [9], it is easy to see that $(\phi, \psi)$ is a weak solution of (2.12) and (2.13). It is known that the equation of $\phi$ can be written as an elliptic system with an anti-symmetric potential $[5,24,30]$ :

$$
\Delta \phi=\Omega \cdot \nabla \phi+f,
$$

with $\Omega \in L^{2}\left(B_{1}, \operatorname{so}(N) \otimes \mathbb{R}^{2}\right)$ satisfying $|\Omega| \leq C\left(|\nabla \phi|+|\psi|^{2}\right)$. Then it follows from Riviere's regularity result and its extensions (see [26-28]) that $\phi \in W^{2, p}(D)$ for any $1<$ $p<2$ which implies $\phi \in W^{1,4}(D)$. Applying a simple argument to the Dirac equation for $\psi$ gives that $\psi \in W^{1,2}(D)$. This indicate that $\psi \in L^{8}(D)$. Then by (2.12), we have $\Delta \phi \in L^{2}(D)$ which implies the conclusion of the theorem. 
In the end of this section, we recall two lemmas which are used in this paper.

Lemma 2.6 ([5]) For any $\psi, \omega \in W^{1,3 / 4}\left(M, \Sigma M \otimes \phi^{\star} T N\right)$, we have

$$
\int_{M}\langle\psi, \not D \omega\rangle=\int_{M}\langle\not D \psi, \omega\rangle-\int_{\partial M}\langle\vec{n} \cdot \psi, \omega\rangle
$$

where $\langle\psi, \omega\rangle:=h_{i j}\left\langle\psi^{i}, \omega^{j}\right\rangle$.

Proposition 2.7 ([2]) Let $N$ be a compact Riemannian manifold. Then there exists a constant $\epsilon_{1}=\epsilon_{1}(N)>0$ such that if $(\phi, \psi)$ is a smooth Dirac-harmonic map from the standard sphere $S^{2}$ to $N$ satisfying

$$
\int_{S^{2}}\left(|d \phi|^{2}+|\psi|^{4}\right)<\epsilon_{1}
$$

then both $\phi$ and $\psi$ are trivial.

\section{Three circle theorem for approximate Dirac-harmonic maps}

In this section, we will extend the three circle theorem for Dirac-harmonic maps in [18] to the case of approximate Dirac-harmonic maps. The idea is from Qing-Tian's paper [22], which used a special case of the three circle theorem due to Simon [25] to show that the tangential energy of the sequence in the neck region decays exponentially. The second author in cooperation with H.Yin has extended this idea to some fourth order equations, see [19,20].

Let us first state the three circle theorem for harmonic functions (see $[18,22,25]$ ).

Theorem 3.1 There exists a constant $L>0$, such that if $u$ is a nontrivial smooth harmonic function defined in $[(i-1) L,(i+2) L] \times S^{1}$ that satisfies

$$
\int_{\{i L\} \times S^{1}} u d \theta=\int_{\{(i+1) L\} \times S^{1}} u d \theta=0,
$$

then

$$
\begin{aligned}
\|u\|_{L^{2}\left([i L,(i+1) L] \times S^{1}\right)}^{2}< & \frac{1}{2}\left(e^{-L}\|u\|_{L^{2}\left([(i-1) L, i L] \times S^{1}\right)}^{2}\right. \\
& \left.+e^{-L}\|u\|_{L^{2}\left([(i+1) L,(i+2) L] \times S^{1}\right)}^{2}\right) .
\end{aligned}
$$

Next, we prove an $L^{2}$ interior estimate for the following integro-differential equations.

Lemma 3.2 Suppose $u \in W^{2,2}\left(D_{4} \backslash D_{1}\right), v \in W^{1,2}\left(D_{4} \backslash D_{1}\right)$ and satisfies

$$
\begin{aligned}
& \Delta u=A^{1} u+A^{2} \nabla u+A^{3} v+\frac{1}{2 \pi} \int_{0}^{2 \pi} A^{4} u+A^{5} \nabla u+A^{6} v d \theta+f_{1}, \\
& \partial \partial=B^{1} u+B^{2} \nabla u+B^{3} v+\frac{1}{2 \pi} \int_{0}^{2 \pi} B^{4} u+B^{5} \nabla u+B^{6} v d \theta+f_{2},
\end{aligned}
$$

where

$$
\sum_{i=1}^{6}\left(\left\|A^{i}\right\|_{L^{4}\left(D_{4} \backslash D_{1}\right)}+\left\|B^{i}\right\|_{L^{4}\left(D_{4} \backslash D_{1}\right)}\right) \leq \rho \quad \text { and } \sum_{i=1}^{2}\left\|f_{i}\right\|_{L^{2}\left(D_{4} \backslash D_{1}\right)} \leq C .
$$


Then there exists a positive constant $\rho_{0}$ such that if $\rho \leq \rho_{0}$, there holds

$$
\begin{aligned}
& \|u\|_{W^{2,2}\left(D_{3} \backslash D_{2}\right)}+\|v\|_{W^{1,2}\left(D_{3} \backslash D_{2}\right)} \\
& \quad \leq C\left(\|u\|_{L^{2}\left(D_{4} \backslash D_{1}\right)}+\|v\|_{L^{2}\left(D_{4} \backslash D_{1}\right)}+\left\|f_{1}\right\|_{L^{2}\left(D_{4} \backslash D_{1}\right)}+\left\|f_{2}\right\|_{L^{2}\left(D_{4} \backslash D_{1}\right)}\right) .
\end{aligned}
$$

Proof The proof is similar to Lemma 3.1 in [18] where $f_{1}=f_{2}=0$.

Denote $B_{\sigma}=D_{3+\sigma} \backslash D_{2-\sigma}, 0<\sigma<1$. Let $\sigma^{\prime}=\frac{\sigma+1}{2}$. Take a cut-off function $\eta(x)=$ $\eta(|x|)$ with compact support in $B_{\sigma^{\prime}}$ satisfying $\eta(x) \equiv 1$ in $B_{\sigma}$ and $|\nabla \eta| \leq \frac{4}{(1-\sigma)}$ and $|\Delta \eta| \leq \frac{16}{(1-\sigma)^{2}}$. Computing directly, we get

$$
\begin{aligned}
\Delta(\eta u)= & \eta \Delta u+2 \nabla \eta \nabla u+\Delta \eta u \\
= & \left(2 \nabla \eta+\eta A^{2}\right) \nabla u+\left(\Delta \eta+\eta A^{1}\right) u+\eta A^{3} v+\eta f_{1} \\
& +\eta \cdot \frac{1}{2 \pi} \int_{0}^{2 \pi} A^{4} u+A^{5} \nabla u+A^{6} v d \theta .
\end{aligned}
$$

By the standard elliptic estimate and Sobolev embedding, we have

$$
\begin{aligned}
\|\eta u\|_{W^{2,2}\left(D_{4}\right)} \leq & C\left(\left\|A^{1} \eta u\right\|_{L^{2}\left(D_{4}\right)}+\left\|A^{2} \eta \nabla u\right\|_{L^{2}\left(D_{4}\right)}\right. \\
& +\left\|A^{3} \eta v\right\|_{L^{2}\left(D_{4}\right)}+\left\|\eta f_{1}\right\|_{L^{2}\left(D_{4}\right)} \\
& +\|\nabla \eta \nabla u\|_{L^{2}\left(D_{4}\right)}+\|\Delta \eta u\|_{L^{2}\left(D_{4}\right)} \\
& \left.+\left\|\eta \cdot \frac{1}{2 \pi} \int_{0}^{2 \pi} A^{4} u+A^{5} \nabla u+A^{6} v d \theta\right\|_{L^{2}\left(D_{4}\right)}\right) \\
\leq & C\left(\left\|A^{1}\right\|_{L^{2}\left(D_{4} \backslash D_{1}\right)}+\left\|A^{4}\right\|_{L^{2}\left(D_{4} \backslash D_{1}\right)}\right. \\
& \left.+\left\|A^{2}\right\|_{L^{4}\left(D_{4} \backslash D_{1}\right)}+\left\|A^{5}\right\|_{L^{4}\left(D_{4} \backslash D_{1}\right)}\right)\|\eta u\|_{W^{2,2}\left(D_{4}\right)} \\
& +C\left(\left\|A^{3}\right\|_{L^{4}\left(D_{4} \backslash D_{1}\right)}+\left\|A^{6}\right\|_{L^{4}\left(D_{4} \backslash D_{1}\right)}\right)\|\eta v\|_{W^{1,2}\left(D_{4}\right)}+C\left(\left\|A^{2} \nabla \eta u\right\|_{L^{2}\left(D_{4}\right)}\right) \\
& \left.+\left\|A^{5} \nabla \eta u\right\|_{L^{2}\left(D_{4}\right)}\right)+C\left(\|\nabla \eta \nabla u\|_{L^{2}\left(D_{4}\right)}+\|\Delta \eta u\|_{L^{2}\left(D_{4}\right)}+\left\|\eta f_{1}\right\|_{L^{2}\left(D_{4}\right)}\right) \\
\leq & C \rho_{0}\|\eta u\|_{W^{2,2}\left(D_{4}\right)}+C \rho_{0}\|\eta v\|_{W^{1,2}\left(D_{4}\right)} \\
& +C\left(\left\|A^{2}\right\|_{L^{4}\left(D_{4} \backslash D_{1}\right)}+\left\|A^{5}\right\|_{L^{4}\left(D_{4} \backslash D_{1}\right)}\right)\|\nabla \eta u\|_{W^{1,2}\left(D_{4}\right)} \\
& +C\left(\frac{\|\nabla u\|_{L^{2}\left(B_{\sigma^{\prime}}\right)}}{1-\sigma u \|_{L^{2}\left(B_{\sigma^{\prime}}\right)}}+\left\|\eta f_{1}\right\|_{L^{2}\left(D_{4}\right)}\right) \\
\leq & C \rho_{0}\left(\|\eta u\|_{W^{2,2}\left(D_{4}\right)}+\|\eta v\|_{W^{1,2}\left(D_{4}\right)}\right) \\
& +C\left(\frac{\|\nabla u\|_{L^{2}\left(B_{\sigma^{\prime}}\right)}}{1-\sigma}+\frac{\|u\|_{L^{2}\left(B_{\sigma^{\prime}}\right)}}{(1-\sigma)^{2}}+\left\|\eta f_{1}\right\|_{L^{2}\left(D_{4}\right)}\right) .
\end{aligned}
$$

Similarly, we can compute

$$
\begin{aligned}
\not \partial(\eta v)= & \eta B^{1} u+\eta B^{2} \nabla u+\left(\eta B^{3}+\nabla \eta\right) v \\
& +\eta \frac{1}{2 \pi} \int_{0}^{2 \pi} B^{4} u+B^{5} \nabla u+B^{6} v d \theta+\eta f_{2} .
\end{aligned}
$$

By the first order elliptic estimate, we have

$$
\begin{aligned}
\|\eta v\|_{W^{1,2}\left(D_{4}\right)} \leq & C\left(\left\|B^{1} \eta u\right\|_{L^{2}\left(D_{4}\right)}+\left\|B^{2} \eta \nabla u\right\|_{L^{2}\left(D_{4}\right)}+\left\|B^{3} \eta v\right\|_{L^{2}\left(D_{4}\right)}\right. \\
& +\left\|\eta f_{2}\right\|_{L^{2}\left(D_{4}\right)}+\|\nabla \eta v\|_{L^{2}\left(D_{4}\right)}
\end{aligned}
$$




$$
\begin{aligned}
& \left.+\left\|\eta \cdot \frac{1}{2 \pi} \int_{0}^{2 \pi} B^{4} u+B^{5} \nabla u+B^{6} v d \theta\right\|_{L^{2}\left(D_{4}\right)}\right) \\
\leq & C\left(\left\|B^{1}\right\|_{L^{4}\left(D_{4} \backslash D_{1}\right)}+\left\|B^{4}\right\|_{L^{4}\left(D_{4} \backslash D_{1}\right)}\right. \\
& \left.+\left\|B^{2}\right\|_{L^{4}\left(D_{4} \backslash D_{1}\right)}+\left\|B^{5}\right\|_{L^{4}\left(D_{4} \backslash D_{1}\right)}\right)\|\eta u\|_{W^{2,2}\left(D_{4}\right)} \\
& +C\left(\left\|B^{3}\right\|_{L^{4}\left(D_{4} \backslash D_{1}\right)}+\left\|B^{6}\right\|_{L^{4}\left(D_{4} \backslash D_{1}\right)}\right)\|\eta v\|_{W^{1,2}\left(D_{4}\right)} \\
& +C\left(\left\|B^{2} \nabla \eta u\right\|_{L^{2}\left(D_{4}\right)}+\left\|B^{5} \nabla \eta u\right\|_{L^{2}\left(D_{4}\right)}\right) \\
& +C\left(\|\nabla \eta v\|_{L^{2}\left(D_{4}\right)}+\left\|\eta f_{2}\right\|_{L^{2}\left(D_{4}\right)}\right) \\
\leq & C \rho_{0}\|\eta u\|_{W^{2,2}\left(D_{4}\right)}+C \rho_{0}\|\eta v\|_{W^{1,2}\left(D_{4}\right)} \\
& +C\left(\left\|B^{2}\right\|_{L^{4}\left(D_{4} \backslash D_{1}\right)}+\left\|B^{5}\right\|_{L^{4}\left(D_{4} \backslash D_{1}\right)}\right)\|\nabla \eta u\|_{W^{1,2}\left(D_{4}\right)} \\
& +C\left(\frac{\|v\|_{L^{2}\left(D_{4} \backslash D_{1}\right)}}{1-\sigma}+\left\|\eta f_{2}\right\|_{L^{2}\left(D_{4}\right)}\right) \\
\leq & C \rho_{0}\left(\|\eta u\|_{W^{2,2}\left(D_{4}\right)}+\|\eta v\|_{W^{1,2}\left(D_{4}\right)}\right) \\
& +C\left(\frac{\|\nabla u\|_{L^{2}\left(B_{\sigma^{\prime}}\right)}}{1-\sigma}+\frac{\|u\|_{L^{2}\left(B_{\sigma^{\prime}}\right)}}{(1-\sigma)^{2}}+\frac{\|v\|_{L^{2}\left(D_{4} \backslash D_{1}\right)}}{1-\sigma}+\left\|f_{2}\right\|_{L^{2}\left(D_{4} \backslash D_{1}\right)}\right) .
\end{aligned}
$$

Taking $\rho_{0}$ sufficiently small, we get

$$
\begin{aligned}
\|\eta u\|_{W^{2,2}\left(B_{1}\right)}+\|\eta v\|_{W^{1,2}\left(B_{1}\right)} \leq & C\left(\frac{\|\nabla u\|_{L^{2}\left(B_{\sigma^{\prime}}\right)}}{1-\sigma}+\frac{\|u\|_{L^{2}\left(B_{\sigma^{\prime}}\right)}}{(1-\sigma)^{2}}+\frac{\|v\|_{L^{2}\left(D_{4} \backslash D_{1}\right)}}{1-\sigma}\right. \\
& \left.+\left\|f_{1}\right\|_{L^{2}\left(D_{4} \backslash D_{1}\right)}+\left\|f_{2}\right\|_{L^{2}\left(D_{4} \backslash D_{1}\right)}\right) .
\end{aligned}
$$

We now introduce seminorms, and define for $j=0,1,2$

$$
\Xi_{j}=\sup _{0 \leq \sigma \leq 1}(1-\sigma)^{j}\left\|D^{j} u\right\|_{L^{2}\left(B_{\sigma}\right)} .
$$

Multiplying (3.6) by $(1-\sigma)^{2}$ and noting that $1-\sigma^{\prime}=\frac{1-\sigma}{2}$, we have

$$
\Xi_{2} \leq C\left(\Xi_{1}+\Xi_{0}+\|v\|_{L^{2}\left(D_{4} \backslash D_{1}\right)}+\left\|f_{1}\right\|_{L^{2}\left(D_{4} \backslash D_{1}\right)}+\left\|f_{2}\right\|_{L^{2}\left(D_{4} \backslash D_{1}\right)}\right) .
$$

We claim now that $\Xi_{j}$ satisfy an interpolation inequality

$$
\Xi_{1} \leq \epsilon \Xi_{2}+\frac{C}{\epsilon} \Xi_{0}
$$

for any $\epsilon>0$, where $C>0$ is a universal constant. In fact, by the definition of $\Xi_{1}$, for any $\gamma>0$, we have

$$
\begin{aligned}
\Xi_{1} & \leq\left(2-\sigma_{\gamma}\right)\|D u\|_{L^{2}\left(B_{\sigma_{\gamma}}\right)}+\gamma \\
& \leq \epsilon\left(2-\sigma_{\gamma}\right)^{2}\left\|D^{2} u\right\|_{L^{2}\left(B_{\sigma_{\gamma}}\right)}+\frac{C}{\epsilon}\|u\|_{L^{2}\left(B_{\sigma_{\gamma}}\right)}+\gamma,
\end{aligned}
$$

where the second inequality is derived from the interpolation Theorem 7.27 (or Theorem 7.28) in [8].

By letting $\gamma \rightarrow 0$, we obtain (3.8). Using (3.8) in (3.7), we then obtain

$$
\Xi_{2} \leq C\left(\|u\|_{L^{2}\left(D_{4} \backslash D_{1}\right)}+\|v\|_{L^{2}\left(D_{4} \backslash D_{1}\right)}+\left\|f_{1}\right\|_{L^{2}\left(D_{4} \backslash D_{1}\right)}+\left\|f_{2}\right\|_{L^{2}\left(D_{4} \backslash D_{1}\right)}\right),
$$


this is

$$
\begin{aligned}
\left\|D^{2} u\right\|_{L^{2}\left(B_{\sigma}\right)} \leq & \frac{C}{(1-\sigma)^{2}}\left(\|u\|_{L^{2}\left(D_{4} \backslash D_{1}\right)}+\|v\|_{L^{2}\left(D_{4} \backslash D_{1}\right)}\right) \\
& \left.+\left\|f_{1}\right\|_{L^{2}\left(D_{4} \backslash D_{1}\right)}+\left\|f_{2}\right\|_{L^{2}\left(D_{4} \backslash D_{1}\right)}\right) .
\end{aligned}
$$

Taking $\sigma=\frac{1}{2}$, it follows

$$
\begin{aligned}
\|u\|_{W^{2,2}\left(B_{1 / 2}\right)} \leq & C\left(\|u\|_{L^{2}\left(D_{4} \backslash D_{1}\right)}+\|v\|_{L^{2}\left(D_{4} \backslash D_{1}\right)}\right. \\
& \left.+\left\|f_{1}\right\|_{L^{2}\left(D_{4} \backslash D_{1}\right)}+\left\|f_{2}\right\|_{L^{2}\left(D_{4} \backslash D_{1}\right)}\right) .
\end{aligned}
$$

Choosing a new cut-off function $\eta$ in (3.6) and using (3.9), we get

$$
\begin{aligned}
\|v\|_{W^{1,2}\left(B_{1 / 4}\right)} \leq & C\left(\|u\|_{L^{2}\left(D_{4} \backslash D_{1}\right)}+\|v\|_{L^{2}\left(D_{4} \backslash D_{1}\right)}\right. \\
& \left.+\left\|f_{1}\right\|_{L^{2}\left(D_{4} \backslash D_{1}\right)}+\left\|f_{2}\right\|_{L^{2}\left(D_{4} \backslash D_{1}\right)}\right) .
\end{aligned}
$$

Then it is easy to see that the lemma follows from (3.9) and (3.10).

Denote $P_{i}:=D_{e^{(i+1) L_{r_{2}}}} \backslash D_{e^{i L_{r_{2}}}}$ and

$$
F_{i}(u, v):=\int_{P_{i}} \frac{1}{|x|^{2}}|u|^{2} d x+\int_{P_{i}} \frac{1}{|x|}|v|^{2} d x,
$$

where $L>0$ is the constant in Theorem 3.1.

We have the following three circle theorem:

Theorem 3.3 Suppose $u \in W^{2,2}\left(P_{i-1} \cup P_{i} \cup P_{i+1}\right), v \in W^{1,2}\left(P_{i-1} \cup P_{i} \cup P_{i+1}\right)$ satisfy equations (3.2) and (3.3). Then there exists a positive constant $\rho_{0}$, such that if $0<\rho_{1}<\rho_{0}$ and

$$
\max _{i-1, i, i+1}\left(\left\||x| f_{1}\right\|_{L^{2}\left(P_{j}\right)}^{2}+\left\||x|^{\frac{1}{2}} f_{2}\right\|_{L^{2}\left(P_{j}\right)}^{2}\right) \leq \rho_{1} F_{i}(u, v),
$$

and for any $e^{(i-1) L} r_{2} \leq r \leq \frac{1}{2} e^{(i+2) L} r_{2}$, there hold

$$
\begin{aligned}
& \left\||x|^{\frac{3}{2}}\left(\left|A^{1}\right|+\left|A^{4}\right|\right)\right\|_{L^{4}\left(D_{2 r} \backslash D_{r}\right)} \\
& \quad+\left\||x|\left(\left|A^{3}\right|+\left|A^{6}\right|+\left|B^{1}\right|+\left|B^{4}\right|\right)\right\|_{L^{4}\left(D_{2 r} \backslash D_{r}\right)} \\
& \quad+\left\||x|^{\frac{1}{2}}\left(\left|A^{2}\right|+\left|A^{5}\right|+\left|B^{3}\right|+\left|B^{6}\right|\right)\right\|_{L^{4}\left(D_{2 r} \backslash D_{r}\right)} \\
& \quad+\left\|\left|B^{2}\right|+\left|B^{5}\right|\right\|_{L^{4}\left(D_{2 r} \backslash D_{r}\right)} \leq \rho_{1},
\end{aligned}
$$

and

$$
\begin{aligned}
& \left|\int_{0}^{2 \pi} u\left(e^{i L} r_{2}, \theta\right) d \theta\right|^{2}+\left|\int_{0}^{2 \pi} u\left(e^{(i+1) L} r_{2}, \theta\right) d \theta\right|^{2} \\
& +\left|\int_{0}^{2 \pi} v\left(e^{i L} r_{2}, \theta\right) d \theta\right|^{2}+\left|\int_{0}^{2 \pi} v\left(e^{(i+1) L} r_{2}, \theta\right) d \theta\right|^{2} \leq \rho_{1} F_{i}(u, v)
\end{aligned}
$$

then, there hold

(a) $F_{i+1}(u, v) \leq e^{-L} F_{i}(u, v)$ implies $F_{i}(u, v) \leq e^{-L} F_{i-1}(u, v)$;

(b) $F_{i-1}(u, v) \leq e^{-L} F_{i}(u, v)$ implies $F_{i}(u, v) \leq e^{-L} F_{i+1}(u, v)$;

(c) either $F_{i}(u, v) \leq e^{-L} F_{i-1}(u, v)$ or $F_{i}(u, v) \leq e^{-L} F_{i+1}(u, v)$. 
Proof Since $F_{i}(u, v),(3.11)$ and (3.12) are scaling invariant, we may assume $r_{2}=1$ and $i=2$. If the conclusion does not hold, there exist sequences $\rho_{1 k} \rightarrow 0, A_{k}^{j}, B_{k}^{j}(\mathrm{j}=1, \ldots, 6)$, $f_{1 k}, f_{2 k}, u_{k}, v_{k}$ such that $u_{k}$ and $v_{k}$ satisfy

$$
\begin{aligned}
\Delta u_{k}= & A_{k}^{1} u_{k}+A_{k}^{2} \nabla u_{k}+A_{k}^{3} v_{k} \\
& +\frac{1}{2 \pi} \int_{0}^{2 \pi} A_{k}^{4} u_{k}+A_{k}^{5} \nabla u_{k}+A_{k}^{6} v_{k} d \theta+f_{1 k}, \\
\not \partial v_{k}= & B_{k}^{1} u_{k}+B_{k}^{2} \nabla u_{k}+B_{k}^{3} v_{k} \\
& +\frac{1}{2 \pi} \int_{0}^{2 \pi} B_{k}^{4} u_{k}+B_{k}^{5} \nabla u_{k}+B_{k}^{6} v_{k} d \theta+f_{2 k},
\end{aligned}
$$

and

$$
\begin{aligned}
& \left|\int_{0}^{2 \pi} u_{k}\left(e^{i L}, \theta\right) d \theta\right|^{2}+\left|\int_{0}^{2 \pi} u_{k}\left(e^{(i+1) L}, \theta\right) d \theta\right|^{2} \\
& \quad+\left|\int_{0}^{2 \pi} u_{k}\left(e^{i L}, \theta\right) d \theta\right|^{2}+\left|\int_{0}^{2 \pi} u_{k}\left(e^{(i+1) L}, \theta\right) d \theta\right|^{2} \leq \rho_{1 k} F_{i}\left(u_{k}, v_{k}\right) .
\end{aligned}
$$

Moreover, for any $e^{(i-1) L} \leq r \leq e^{(i+2) L}, A_{k}^{j}, B_{k}^{j}, f_{1 k}, f_{2 k}$ satisfy

$$
\begin{aligned}
& \left\||x|^{\frac{3}{2}}\left(\left|A_{k}^{1}\right|+\left|A_{k}^{4}\right|\right)\right\|_{L^{4}\left(D_{2 r} \backslash D_{r}\right)} \\
& \quad+\left\||x|\left(\left|A_{k}^{3}\right|+\left|A_{k}^{6}\right|+\left|B_{k}^{1}\right|+\left|B_{k}^{4}\right|\right)\right\|_{L^{4}\left(D_{2 r} \backslash D_{r}\right)} \\
& \quad+\left\||x|^{\frac{1}{2}}\left(\left|A_{k}^{2}\right|+\left|A_{k}^{5}\right|+\left|B_{k}^{3}\right|+\left|B_{k}^{6}\right|\right)\right\|_{L^{4}\left(D_{2 r} \backslash D_{r}\right)} \\
& \quad+\left\|\left|B_{k}^{2}\right|+\left|B_{k}^{5}\right|\right\|_{L^{4}\left(D_{2 r} \backslash D_{r}\right)} \leq \rho_{1 k}
\end{aligned}
$$

and

$$
\max _{i-1, i, i+1}\left(\left\||x| f_{1 k}\right\|_{L^{2}\left(P_{j}\right)}^{2}+\left\||x|^{\frac{1}{2}} f_{2 k}\right\|_{L^{2}\left(P_{j}\right)}^{2}\right) \leq \rho_{1 k} F_{i}(u, v) .
$$

But, $u_{k}$ does not satisfy at least one of the conclusions in $(a),(b)$ and $(c)$.

If $(a)$ does not hold, then we have

$$
F_{2}\left(u_{k}, v_{k}\right) \geq e^{L} F_{3}\left(u_{k}, v_{k}\right) \quad \text { and } \quad F_{2}\left(u_{k}, v_{k}\right) \geq e^{-L} F_{1}\left(u_{k}, v_{k}\right) ;
$$

If $(b)$ does not hold, then we have

$$
F_{2}\left(u_{k}, v_{k}\right) \geq e^{L} F_{1}\left(u_{k}, v_{k}\right) \quad \text { and } \quad F_{2}\left(u_{k}, v_{k}\right) \geq e^{-L} F_{3}\left(u_{k}, v_{k}\right) ;
$$

If (c) does not hold, then we have

$$
F_{2}\left(u_{k}, v_{k}\right) \geq e^{-L} \max \left\{F_{1}\left(u_{k}, v_{k}\right), F_{3}\left(u_{k}, v_{k}\right)\right\} ;
$$

In all those three cases, we may get the same conclusion that

$$
2 F_{2}\left(u_{k}, v_{k}\right) \geq e^{-L}\left(F_{1}\left(u_{k}, v_{k}\right)+F_{3}\left(u_{k}, v_{k}\right)\right) .
$$

Without loss of generality, we assume $F_{2}\left(u_{k}, v_{k}\right)=1$ (if not, we consider $\tilde{u_{k}}=\frac{u_{k}}{F_{2}\left(u_{k}, v_{k}\right)}$ and $\left.\widetilde{v_{k}}=\frac{v_{k}}{F_{2}\left(u_{k}, v_{k}\right)}\right)$. Then we obtain

$$
\left\|u_{k}\right\|_{L^{2}\left(P_{1} \cup P_{2} \cup P_{3}\right)}+\left\|v_{k}\right\|_{L^{2}\left(P_{1} \cup P_{2} \cup P_{3}\right)} \leq C .
$$


By Lemma 3.2, we have $\left\|u_{k}\right\|_{W^{2,2}\left(P_{2}\right)}+\left\|v_{k}\right\|_{W^{1,2}\left(P_{2}\right)} \leq C$. So, there exists a subsequence of $\left(u_{k}, v_{k}\right)$ (we still denote it by $\left(u_{k}, v_{k}\right)$ ), such that

$$
\begin{aligned}
& u_{k} \rightarrow u, \quad v_{k} \rightarrow v \text { weakly in } L^{2}\left(P_{1} \cup P_{2} \cup P_{3}\right) ; \\
& u_{k} \rightarrow u, \quad v_{k} \rightarrow v \text { strongly in } L^{2}\left(P_{2}\right) .
\end{aligned}
$$

It is easy to see that $u$ is a harmonic function and $v$ is a holomorphic function in $D_{e^{(i+2) L} \backslash D_{e^{(i-1) L}} \text { and they satisfy }}$

$$
\int_{\partial D_{e^{i L}}} u=\int_{\partial D_{e^{(i+1) L}}} u=\int_{\partial D_{e^{i L}}} v=\int_{\partial D_{e^{(i+1) L}}} v=0 .
$$

Let $f: \mathbb{R}^{1} \times \mathbb{S}^{1} \rightarrow \mathbb{R}^{2}, f(t, \theta)=\left(e^{t}, \theta\right)(t, \theta) \in \mathbb{R}^{1} \times \mathbb{S}^{1}$ where $\mathbb{R}^{1} \times \mathbb{S}^{1}$ is equipped with the metric $g=d t^{2}+d \theta^{2}$, which is conformal to the standard Euclidean metric $d s^{2}$ on $\mathbb{R}^{2}$. In fact,

$$
\left(f^{-1}\right)^{*} g=\frac{1}{r^{2}} d s^{2}
$$

Then we know that $u \circ f$ is a harmonic function and $e^{\frac{t}{2}} v \circ f$ is a holomorphic function in $[L, 4 L] \times S^{1}$.

According to Theorem 3.1, we know

$$
\begin{aligned}
& \|u \circ f\|_{L^{2}\left([2 L, 3 L] \times S^{1}\right)}^{2} \\
& \quad<\frac{1}{2}\left(e^{-L}\|u \circ f\|_{L^{2}\left([L, 2 L] \times S^{1}\right)}^{2}+e^{-L}\|u \circ f\|_{L^{2}\left([3 L, 4 L] \times S^{1}\right)}^{2}\right)
\end{aligned}
$$

and

$$
\begin{aligned}
& \left\|e^{\frac{t}{2}} v \circ f\right\|_{L^{2}\left([2 L, 3 L] \times S^{1}\right)}^{2} \\
& \quad<\frac{1}{2}\left(e^{-L}\left\|e^{\frac{t}{2}} v \circ f\right\|_{L^{2}\left([L, 2 L] \times S^{1}\right)}^{2}+e^{-L}\left\|e^{\frac{t}{2}} v \circ f\right\|_{L^{2}\left([3 L, 4 L] \times S^{1}\right)}^{2}\right)
\end{aligned}
$$

which implies

$$
\left\|\frac{1}{|x|} u\right\|_{L^{2}\left(P_{2}\right)}^{2}<\frac{1}{2}\left(e^{-L}\left\|\frac{1}{|x|} u\right\|_{L^{2}\left(P_{1}\right)}^{2}+e^{-L}\left\|\frac{1}{|x|} u\right\|_{L^{2}\left(P_{3}\right)}^{2}\right)
$$

and

$$
\left\|\frac{1}{\sqrt{|x|}} v\right\|_{L^{2}\left(P_{2}\right)}^{2}<\frac{1}{2}\left(e^{-L}\left\|\frac{1}{\sqrt{|x|}} v\right\|_{L^{2}\left(P_{1}\right)}^{2}+e^{-L}\left\|\frac{1}{\sqrt{|x|}} v\right\|_{L^{2}\left(P_{3}\right)}^{2}\right) .
$$

Thus,

$$
2 F_{2}(u, v)<e^{-L}\left(F_{1}(u, v)+F_{3}(u, v)\right) .
$$

But, letting $k \rightarrow \infty$ in (3.13) which implies

$$
2 F_{2}(u, v) \geq e^{-L}\left(F_{1}(u, v)+F_{3}(u, v)\right) .
$$

This contradiction finishes the proof.

As a direct corollary of the three circle theorem, we can get the following decay lemma. 
Lemma 3.4 Let $\rho_{1}>0$ be the constant in Theorem 3.3. Let $u \in W^{2,2}\left(D_{e^{(l+1) L_{r_{2}}}} \backslash D_{r_{2}}\right), v \in$

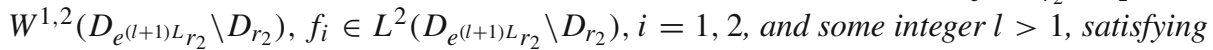
equations (3.2), (3.3) and for any $r_{2} \leq r \leq \frac{1}{2} e^{(l+1) L} r_{2}$, there hold

$$
\begin{aligned}
& \left\||x|^{\frac{3}{2}}\left(\left|A^{1}\right|+\left|A^{4}\right|\right)\right\|_{L^{4}\left(D_{2 r} \backslash D_{r}\right)} \\
& \quad+\left\||x|\left(\left|A^{3}\right|+\left|A^{6}\right|+\left|B^{1}\right|+\left|B^{4}\right|\right)\right\|_{L^{4}\left(D_{2 r} \backslash D_{r}\right)} \\
& \quad+\left\||x|^{\frac{1}{2}}\left(\left|A^{2}\right|+\left|A^{5}\right|+\left|B^{3}\right|+\left|B^{6}\right|\right)\right\|_{L^{4}\left(D_{2 r} \backslash D_{r}\right)} \\
& \quad+\left\|\left|B^{2}\right|+\left|B^{5}\right|\right\|_{L^{4}\left(D_{2 r} \backslash D_{r}\right)} \leq \rho_{1},
\end{aligned}
$$

and

$$
\int_{\partial D_{r}} u=\int_{\partial D_{r}} v=0
$$

Then we have

$$
F_{i}(u, v) \leq C\left(e^{l L} r_{2}+F_{0}(u, v)+F_{l}(u, v)\right)\left(e^{-(l-i) L}+e^{-i L}\right) .
$$

Proof Denote the set of $j(0<j<l)$ for which

$$
\max _{j-1, j, j+1}\left(\left\||x| f_{1}\right\|_{L^{2}\left(P_{i}\right)}^{2}+\left\||x|^{\frac{1}{2}} f_{2}\right\|_{L^{2}\left(P_{i}\right)}^{2}\right)>\rho_{1} F_{j}(u, v)
$$

by $J:=\left\{j_{1}, \ldots, j_{k}\right\}$. If $J=\emptyset$, according to $(c)$ of Theorem 3.3, we get

$$
F_{i}(u, v) \leq e^{-L} F_{i-1}(u, v) \text { or } F_{i}(u, v) \leq e^{-L} F_{i+1}(u, v) .
$$

Then using the $(a)$ and $(b)$ of Theorem 3.3, by iterating, we obtain

$$
F_{i}(u, v) \leq e^{-L i} F_{0}(u, v) \text { or } F_{i}(u, v) \leq e^{-L(l-i)} F_{l}(u, v) .
$$

So, we have

$$
F_{i}(u, v) \leq \frac{1}{2}\left(e^{-L i} F_{0}(u, v)+e^{-L(l-i)} F_{l}(u, v)\right),
$$

which implies (3.17) immediately.

If $J \neq \emptyset$, without loss of generality, we may assume

$$
0<j_{1}<j_{2}<\cdots<j_{k}<l .
$$

Then for each $j_{m}, m=1, \ldots, k$, we have

$$
\begin{aligned}
F_{j_{m}}(u, v) & \leq C \max _{j_{m}-1, j_{m}, j_{m}+1}\left(\left\||x| f_{1}\right\|_{L^{2}\left(P_{i}\right)}^{2}+\left\||x|^{\frac{1}{2}} f_{2}\right\|_{L^{2}\left(P_{i}\right)}^{2}\right) \\
& \leq C e^{j_{m} L} r_{2}=C\left(e^{l L} r_{2}\right) e^{-L\left(l-j_{m}\right)} .
\end{aligned}
$$

By the choice of $j_{m}$, the condition (3.11) holds for $j_{m}<i<j_{m+1}, m=1, \ldots, k-1$. Similar to deriving (3.19), we obtain

$$
\begin{aligned}
F_{i}(u, v) & \leq e^{-L\left(i-j_{m}\right)} F_{j_{m}}(u, v) \text { or } F_{i}(u, v) \\
& \leq e^{-L\left(j_{m+1}-i\right)} F_{j_{m+1}}(u, v) .
\end{aligned}
$$

Thus, we know for $j_{1} \leq i \leq j_{k}$, there exists $m \in\{1, \ldots, k-1\}$ such that $j_{m} \leq i \leq j_{m+1}$. Then we get 


$$
\begin{aligned}
F_{i}(u, v) & \leq \frac{1}{2}\left(e^{-L\left(i-j_{m}\right)} F_{j_{m}}(u, v)+e^{-L\left(j_{m+1}-i\right)} F_{j_{m+1}}(u, v)\right) \\
& \leq C\left(e^{l L} r_{2}\right)\left(e^{-L\left(i-j_{m}\right)} e^{-L\left(l-j_{m}\right)}+e^{-L\left(j_{m+1}-i\right)} e^{-L\left(l-j_{m+1}\right)}\right) \\
& \leq C\left(e^{l L} r_{2}\right) e^{-(l-i) L} .
\end{aligned}
$$

So, if $j_{1}=1$ and $j_{k}=l-1$, then the inequality (3.19) follows immediately. If not, assuming $j_{1}>1$, similar to deriving (3.20), we have, for $1 \leq i \leq j_{1}$,

$$
\begin{aligned}
F_{i}(u, v) & \leq \frac{1}{2}\left(e^{-L i} F_{0}(u, v)+e^{-L\left(j_{1}-i\right)} F_{j_{1}}(u, v)\right) \\
& \leq C\left(e^{-L i} F_{0}(u, v)+\left(e^{l L} r_{2}\right) e^{-(l-i) L}\right) .
\end{aligned}
$$

Similarly, if $j_{k}<l-1$, then for $j_{k} \leq i \leq l$, we have

$$
\begin{aligned}
F_{i}(u, v) & \leq \frac{1}{2}\left(e^{-L(l-i)} F_{l}(u, v)+e^{-L\left(i-j_{k}\right)} F_{j_{k}}(u, v)\right) \\
& \leq C\left(e^{-L(l-i)} F_{l}(u, v)+\left(e^{l L} r_{2}\right) e^{-(l-i) L}\right) .
\end{aligned}
$$

Combining the preceding estimates proves the lemma.

Corollary 3.5 Under the assumptions of Lemma 3.4, we have

$$
\begin{aligned}
& \|\nabla u\|_{L^{2}\left(P_{i}\right)}+\|\nabla v\|_{L^{\frac{4}{3}}\left(P_{i}\right)} \\
& \leq C\left(\left(e^{l L} r_{2}\right)^{\frac{1}{2}}+F_{0}^{1 / 2}(u, v)+F_{l}^{1 / 2}(u, v)\right)\left(e^{-\frac{1}{2}(l-i) L}+e^{-\frac{1}{2} i L}\right) .
\end{aligned}
$$

Proof By Lemma 3.2, Lemma 3.4 and a standard scaling argument, we get

$$
\begin{aligned}
\|\nabla u\|_{L^{2}\left(P_{i}\right)}+\|\nabla v\|_{L^{\frac{4}{3}\left(P_{i}\right)}} \leq & C\left(F_{i-1}^{1 / 2}(u, v)+F_{i}^{1 / 2}(u, v)\right. \\
& +F_{i+1}^{1 / 2}(u, v)+e^{i L} r_{2}\left\|f_{1}\right\|_{L^{2}\left(P_{i-1} \cup P_{i} \cup P_{i+1}\right)} \\
& \left.+\left(e^{i L} r_{2}\right)^{\frac{1}{2}}\left\|f_{2}\right\|_{L^{2}\left(P_{i-1} \cup P_{i} \cup P_{i+1}\right)}\right) \\
\leq & C\left(\left(e^{l L} r_{2}\right)^{\frac{1}{2}}+F_{0}^{1 / 2}(u)+F_{l}^{1 / 2}(u)\right)\left(e^{-\frac{1}{2}(l-i) L}+e^{-\frac{1}{2} i L}\right) .
\end{aligned}
$$

\section{Energy identity and no neck result}

In this section, we will prove our main result Theorem 1.2.

We first consider the following simpler case of a single interior blow-up point.

Theorem 4.1 Let $\left(\phi_{n}, \psi_{n}\right)$ be a sequence of smooth approximate Dirac-harmonic maps from $D_{1}(0)$ to $N$ with

(a) $\left\|\phi_{n}\right\|_{W^{1,2}(D)}+\left\|\psi_{n}\right\|_{L^{4}(D)}+\left\|\tau_{n}\right\|_{L^{2}(D)}+\left\|h_{n}\right\|_{L^{4}(D)} \leq \Lambda$,

(b) $\left(\phi_{n}, \psi_{n}\right) \rightarrow(\phi, \psi)$ strongly in $W_{l o c}^{1,2}\left(D \backslash\{0\}, \mathbb{R}^{N}\right) \times W_{l o c}^{1, \frac{4}{3}}\left(D \backslash\{0\}, \mathbb{R}^{N}\right)$ as $n \rightarrow \infty$, 
where $\tau_{n}:=\tau\left(\phi_{n}, \psi_{n}\right)$ and $h_{n}:=h\left(\phi_{n}, \psi_{n}\right)$. Then there exist a subsequence of $\left(\phi_{n}, \psi_{n}\right)($ still denoted by $\left.\left(\phi_{n}, \psi_{n}\right)\right)$ and a nonnegative integer $Q$ such that, for any $i=1, \ldots, Q$, there exist point $x_{n}^{i}$, positive numbers $\lambda_{n}^{i}$ and a nonconstant Dirac-harmonic sphere $\left(\sigma^{i}, \xi^{i}\right): S^{2} \rightarrow N$ such that:

(1) $x_{n}^{i} \rightarrow 0, \lambda_{n}^{i} \rightarrow 0$, as $n \rightarrow \infty$;

(2) $\lim _{n \rightarrow \infty}\left(\frac{\lambda_{n}^{i}}{\lambda_{n}^{j}}+\frac{\lambda_{n}^{j}}{\lambda_{n}^{i}}+\frac{\left|x_{n}^{i}-x_{n}^{j}\right|}{\lambda_{n}^{i}+\lambda_{n}^{j}}\right)=\infty$ for any $i \neq j$;

(3) $\left(\sigma^{i}, \xi^{i}\right)$ is the weak limit of $\left(\phi_{n}\left(x_{n}^{i}+\lambda_{n}^{i} x\right), \sqrt{\lambda_{n}^{i}} \psi_{n}\left(x_{n}^{i}+\lambda_{n}^{i} x\right)\right)$ in $W_{l o c}^{1,2}\left(\mathbb{R}^{2}\right) \times W_{l o c}^{1, \frac{4}{3}}\left(\mathbb{R}^{2}\right)$;

(4) Energy identity: we have

$$
\begin{aligned}
& \lim _{n \rightarrow \infty} E\left(\phi_{n}\right)=E(\phi)+\sum_{i=1}^{Q} E\left(\sigma^{i}\right), \\
& \lim _{n \rightarrow \infty} E\left(\psi_{n}\right)=E(\psi)+\sum_{i=1}^{Q} E\left(\xi^{i}\right),
\end{aligned}
$$

(5) No neck property: The image

$$
\phi(D) \cup \bigcup_{i=1}^{Q} \sigma^{i}\left(S^{2}\right)
$$

is a connected set.

Proof Assume 0 is the only blow-up point of the sequence $\left\{\left(\phi_{n}, \psi_{n}\right)\right\}$ in $D$, i.e.

$$
\liminf _{n \rightarrow \infty} E\left(\phi_{n}, \psi_{n} ; D_{r}\right) \geq \frac{\epsilon_{0}^{2}}{2} \text { for all } r>0 .
$$

By the standard argument of blow-up analysis we can assume that, for any $n$, there exist sequences $x_{n} \rightarrow 0$ and $r_{n} \rightarrow 0$ such that

$$
E\left(\phi_{n}, \psi_{n} ; D_{r_{n}}\left(x_{n}\right)\right)=\sup _{\substack{x \in D, r \leq r_{n} \\ D_{r}(x) \subset D}} E\left(\phi_{n}, \psi_{n} ; D_{r}(x)\right)=\frac{\epsilon_{0}^{2}}{4} .
$$

Denoting

$$
\phi_{n}^{\prime}(x):=\phi_{n}\left(x_{n}+r_{n} x\right), \psi_{n}^{\prime}(x):=\sqrt{r_{n}} \psi_{n}\left(x_{n}+r_{n} x\right)
$$

then we have

$$
\begin{gathered}
\tau\left(\phi_{n}^{\prime}, \psi_{n}^{\prime}\right)=\Delta \phi_{n}^{\prime}-A\left(d \phi_{n}^{\prime}, d \phi_{n}^{\prime}\right)-\operatorname{Re}\left(P\left(\mathcal{A}\left(d \phi_{n}^{\prime}\left(e_{\alpha}\right), e_{\alpha} \cdot \psi_{n}^{\prime}\right) ; \psi_{n}^{\prime}\right)\right), \\
h\left(\phi_{n}^{\prime}, \psi_{n}^{\prime}\right)=\not \partial \psi_{n}^{\prime}-\mathcal{A}\left(d \phi_{n}^{\prime}\left(e_{\alpha}\right), e_{\alpha} \cdot \psi_{n}^{\prime}\right),
\end{gathered}
$$

where $\tau\left(\phi_{n}^{\prime}, \psi_{n}^{\prime}\right)=r_{n}^{2} \tau\left(\phi_{n}, \psi_{n}\right)$ and $h\left(\phi_{n}^{\prime}, \psi_{n}^{\prime}\right)=r_{n}^{3 / 2} h\left(\phi_{n}, \psi_{n}\right)$. Noting that for any $D_{R}(y) \subset \mathbb{R}^{2}$ with $R>0$, there holds

$$
E\left(\phi_{n}^{\prime}, \psi_{n}^{\prime} ; D_{R}(y)\right) \leq E\left(\phi_{n}, \psi_{n} ; D_{\frac{1}{2}}\left(x_{n}\right)\right) \leq \Lambda<\infty,
$$




$$
E\left(\phi_{n}^{\prime}, \psi_{n}^{\prime} ; D\right)=E\left(\phi_{n}, \psi_{n} ; D_{r_{n}}\left(x_{n}\right)\right)=\frac{\epsilon_{0}^{2}}{4},
$$

for $n$ large enough. By the small energy regularity Theorem 2.1, the removable singularity Theorem 2.5 and conformal invariance of Dirac-harmonic maps in dimension two, we can take a subsequence, still denoted by $\left(\phi_{n}^{\prime}, \psi_{n}^{\prime}\right)$, that strongly converges to a nonconstant Diracharmonic sphere. This is the first bubble.

By the standard induction argument in [7], we only need to prove the theorem in the case where there is only one bubble. Under this assumption, we have the following:

Claim For any $\epsilon>0$, there exist $\delta>0$ and $R>0$ such that

$$
E\left(\phi_{n}, \psi_{n} ; D_{8 t}\left(x_{n}\right) \backslash D_{t}\left(x_{n}\right)\right) \leq \epsilon^{2} \text { for any } t \in\left(\frac{1}{2} r_{n} R, 2 \delta\right)
$$

when $n$ is large enough.

Proof In fact, if (4.9) is not true, then we can find $\bar{\epsilon}>0, t_{n} \rightarrow 0$, such that $\lim _{n \rightarrow \infty} \frac{t_{n}}{r_{n}}=\infty$ and

$$
E\left(\phi_{n}, \psi_{n} ; D_{8 t_{n}}\left(x_{n}\right) \backslash D_{t_{n}}\left(x_{n}\right)\right) \geq \bar{\epsilon}>0 .
$$

Setting

$$
u_{n}(x):=\phi_{n}\left(x_{n}+t_{n} x\right), v_{n}(x):=\sqrt{t_{n}} \psi_{n}\left(x_{n}+t_{n} x\right),
$$

then it is easy to see that 0 is an energy concentration point for $\left(u_{n}, v_{n}\right)$. We have to consider the following two cases:

(a) $\left(u_{n}, v_{n}\right)$ has no other energy concentration points except 0 .

By Theorem 2.1, passing to a subsequence, we may assume that $\left(u_{n}, v_{n}\right)$ converges to a Dirac-harmonic map $(\sigma, \xi): \mathbb{R}^{2} \rightarrow N$ strongly in $W_{l o c}^{1,2}\left(\mathbb{R}^{2}\right) \times L_{l o c}^{4}\left(\mathbb{R}^{2}\right)$ as $n \rightarrow \infty$. In particular, we have

$$
\lim _{n \rightarrow \infty} E\left(u_{n}, v_{n} ; D_{8} \backslash D_{1}\right)=E\left(\sigma, \xi ; D_{8} \backslash D_{1}\right) \geq \bar{\epsilon} .
$$

According to the standard theory of Dirac-harmonic maps, we know that $(\sigma, \xi)$ is a nontrivial Dirac-harmonic sphere. This is the second bubble. This is a contradiction to the "one bubble" assumption.

(b) $\left(u_{n}, v_{n}\right)$ has another energy concentration point $p \neq 0$.

Without loss of generality, we may assume that $p$ is the only blow-up point in $D_{r}(p)$ for some small $r>0$. By the standard theory of blow-up analysis, there exist $x_{n}^{\prime} \rightarrow p$ and $r_{n}^{\prime} \rightarrow 0$ such that

$$
E\left(u_{n}, v_{n} ; D_{r_{n}^{\prime}}\left(x_{n}^{\prime}\right)\right)=\sup _{\substack{x \in D_{r}(p), s \leq r_{n} \\ D_{s}(x) \subset D_{r}(p)}} E\left(u_{n}, v_{n} ; D_{s}(x)\right)=\frac{\epsilon_{0}^{2}}{4} .
$$

From the process of constructing the first bubble, we know that there exists a nontrivial Dirac-harmonic sphere $(\widetilde{\sigma}, \widetilde{\xi})$ such that

$$
\left(u_{n}\left(x_{n}^{\prime}+r_{n}^{\prime} x\right), r_{n}^{\prime 1 / 2} v_{n}\left(x_{n}^{\prime}+r_{n}^{\prime} x\right)\right) \rightarrow(\widetilde{\sigma}, \widetilde{\xi}) \text { strongly in } W_{l o c}^{1,2}\left(\mathbb{R}^{2}\right) \times L_{l o c}^{4}\left(\mathbb{R}^{2}\right)
$$

as $n \rightarrow \infty$. This is

$$
\left(\phi_{n}\left(x_{n}+t_{n} x_{n}^{\prime}+t_{n} r_{n}^{\prime} x\right),\left(t_{n} r_{n}^{\prime}\right)^{1 / 2} \psi_{n}\left(x_{n}+t_{n} x_{n}^{\prime}+t_{n} r_{n}^{\prime} x\right)\right) \rightarrow(\widetilde{\sigma}, \widetilde{\xi})
$$




$$
\text { strongly in } W_{l o c}^{1,2}\left(\mathbb{R}^{2}\right) \times L_{l o c}^{4}\left(\mathbb{R}^{2}\right)
$$

as $n \rightarrow \infty$. By (4.11), $(\widetilde{\sigma}, \widetilde{\xi})$ is nontrivial. Therefore, we again get the second bubble contradicting the "one bubble" assumption. So, we proved Claim (4.9).

By Theorem 2.1, for any $t \in\left(r_{n} R, \delta\right)$, we obtain

$$
\begin{aligned}
& \left\||x|^{\frac{3}{4}} \nabla \phi_{n}\right\|_{L^{8}\left(D_{2 t}\left(x_{n}\right) \backslash D_{t}\left(x_{n}\right)\right)}+\left\||x|^{\frac{3}{8}} \psi_{n}\right\|_{L^{16}\left(D_{2 t}\left(x_{n}\right) \backslash D_{t}\left(x_{n}\right)\right)} \\
& \leq C\left(\left\|\nabla \phi_{n}\right\|_{L^{2}\left(D_{4 t}\left(x_{n}\right) \backslash D_{t / 2}\left(x_{n}\right)\right)}+\left\|\psi_{n}\right\|_{L^{4}\left(D_{4 t}\left(x_{n}\right) \backslash D_{t / 2}\left(x_{n}\right)\right)}+t\left\|\tau_{n}\right\|_{L^{2}\left(D_{4 t}\left(x_{n}\right) \backslash D_{t / 2}\left(x_{n}\right)\right)}\right. \\
& \left.\quad+\sqrt{t}\left\|h_{n}\right\|_{L^{2}\left(D_{4 t}\left(x_{n}\right) \backslash D_{t / 2}\left(x_{n}\right)\right)}\right) \\
& \leq C(\epsilon+\sqrt{\delta}) .
\end{aligned}
$$

For simplicity, we will denote $\phi_{n}, \psi_{n}, \tau_{n}, h_{n}$ by $\phi, \psi, \tau$ and $h$ respectively.

We define $\phi^{*}(r)$ and $\psi^{*}(r)$ as follows:

$$
\phi^{*}(r)=\frac{1}{2 \pi} \int_{0}^{2 \pi} \phi(r, \theta) d \theta \text { and } \psi^{*}(r)=\frac{1}{2 \pi} \int_{0}^{2 \pi} \psi(r, \theta) d \theta .
$$

Next, we use the same method as in [18] to compute the equation for $\left(\phi-\phi^{*}, \psi-\psi^{*}\right)$. Here, for reader's convenience, we repeat this process again.

By equation (1.7), we have

$$
\begin{aligned}
\Delta \phi^{*}(t) & =\frac{1}{2 \pi} \int_{0}^{2 \pi} A(\phi)(d \phi, d \phi)+\operatorname{Re}\left(P(\phi)\left(\mathcal{A}(\phi)\left(d \phi\left(e_{\alpha}\right), e_{\alpha} \cdot \psi\right) ; \psi\right)\right)+\tau d \theta \\
& =\mathrm{I}+\mathrm{II}+\frac{1}{2 \pi} \int_{0}^{2 \pi} \tau d \theta .
\end{aligned}
$$

Computing directly, we have

$$
\begin{aligned}
\mathrm{I}= & \frac{1}{2 \pi} \int_{0}^{2 \pi} A(\phi)(d \phi, d \phi)-A\left(\phi^{*}\right)(d \phi, d \phi) \\
& +A\left(\phi^{*}\right)(d \phi, d \phi)-A\left(\phi^{*}\right)\left(d \phi^{*}, d \phi^{*}\right)+A\left(\phi^{*}\right)\left(d \phi^{*}, d \phi^{*}\right) d \theta \\
= & A\left(\phi^{*}\right)\left(d \phi^{*}, d \phi^{*}\right)+\frac{1}{2 \pi} \int_{0}^{2 \pi} A^{4}\left(\phi-\phi^{*}\right)+A^{5} \nabla\left(\phi-\phi^{*}\right) d \theta,
\end{aligned}
$$

and

$$
\begin{aligned}
\mathrm{II}= & \frac{1}{2 \pi} \operatorname{Re} \int_{0}^{2 \pi} P(\phi)\left(\mathcal{A}(\phi)\left(d \phi\left(e_{\alpha}\right), e_{\alpha} \cdot \psi\right) ; \psi\right) \\
& -P\left(\phi^{*}\right)\left(\mathcal{A}(\phi)\left(d \phi\left(e_{\alpha}\right), e_{\alpha} \cdot \psi\right) ; \psi\right) \\
& +P\left(\phi^{*}\right)\left(\mathcal{A}(\phi)\left(d \phi\left(e_{\alpha}\right), e_{\alpha} \cdot \psi\right) ; \psi\right) \\
& -P\left(\phi^{*}\right)\left(\mathcal{A}\left(\phi^{*}\right)\left(d \phi\left(e_{\alpha}\right), e_{\alpha} \cdot \psi\right) ; \psi\right) \\
& +P\left(\phi^{*}\right)\left(\mathcal{A}\left(\phi^{*}\right)\left(d \phi\left(e_{\alpha}\right), e_{\alpha} \cdot \psi\right) ; \psi\right) \\
& -P\left(\phi^{*}\right)\left(\mathcal{A}\left(\phi^{*}\right)\left(d \phi^{*}\left(e_{\alpha}\right), e_{\alpha} \cdot \psi\right) ; \psi\right) \\
& +P\left(\phi^{*}\right)\left(\mathcal{A}\left(\phi^{*}\right)\left(d \phi^{*}\left(e_{\alpha}\right), e_{\alpha} \cdot \psi\right) ; \psi\right) \\
& -P\left(\phi^{*}\right)\left(\mathcal{A}\left(\phi^{*}\right)\left(d \phi^{*}\left(e_{\alpha}\right), e_{\alpha} \cdot \psi^{*}\right) ; \psi\right) \\
& +P\left(\phi^{*}\right)\left(\mathcal{A}\left(\phi^{*}\right)\left(d \phi^{*}\left(e_{\alpha}\right), e_{\alpha} \cdot \psi^{*}\right) ; \psi\right) \\
& -P\left(\phi^{*}\right)\left(\mathcal{A}\left(\phi^{*}\right)\left(d \phi^{*}\left(e_{\alpha}\right), e_{\alpha} \cdot \psi^{*}\right) ; \psi^{*}\right) \\
& +P\left(\phi^{*}\right)\left(\mathcal{A}\left(\phi^{*}\right)\left(d \phi^{*}\left(e_{\alpha}\right), e_{\alpha} \cdot \psi^{*}\right) ; \psi^{*}\right) d \theta
\end{aligned}
$$




$$
\begin{aligned}
= & \operatorname{Re}\left(P\left(\phi^{*}\right)\left(\mathcal{A}\left(\phi^{*}\right)\left(d \phi^{*}\left(e_{\alpha}\right), e_{\alpha} \cdot \Psi^{*}\right) ; \psi^{*}\right)\right) \\
& +\frac{1}{2 \pi} \int_{0}^{2 \pi} A^{4}\left(\phi-\phi^{*}\right)+A^{5} \nabla\left(\phi-\phi^{*}\right) \\
& +\frac{1}{2 \pi} \operatorname{Re} \int_{0}^{2 \pi} A^{6}\left(\psi-\psi^{*}\right) d \theta,
\end{aligned}
$$

where $A^{i}$ may differ from line to line and just stands for an expression satisfying

$$
\begin{aligned}
& \left|A^{4}\right| \leq C(N)\left(|d \phi|^{2}+|d \phi||\psi|^{2}\right), \\
& \left|A^{5}\right| \leq C(N)\left(|d \phi|+|\psi|^{2}\right), \\
& \left|A^{6}\right| \leq C(N)|d \phi||\psi| .
\end{aligned}
$$

Moreover, (4.12) implies

$$
\left\||x|^{\frac{3}{2}} A^{4}\right\|_{L^{4}\left(D_{2 t} \backslash D_{t}\right)}+\left\||x| A^{6}\right\|_{L^{4}\left(D_{2 t} \backslash D_{t}\right)}+\left\||x|^{\frac{1}{2}} A^{5}\right\|_{L^{4}\left(D_{2 t} \backslash D_{t}\right)} \leq C \epsilon
$$

for any $t \in\left(\frac{1}{2} r_{n} R, 2 \delta\right)$. Then, we get

$$
\begin{aligned}
\Delta\left(\phi-\phi^{*}\right)= & A(\phi)(d \phi, d \phi)-A\left(\phi^{*}\right)\left(d \phi^{*}, d \phi^{*}\right) \\
& +\operatorname{ReP}(\phi)\left(\mathcal{A}(\phi)\left(d \phi\left(e_{\alpha}\right), e_{\alpha} \cdot \psi\right) ; \psi\right) \\
& -\operatorname{ReP}\left(\phi^{*}\right)\left(\mathcal{A}\left(\phi^{*}\right)\left(d \phi^{*}\left(e_{\alpha}\right), e_{\alpha} \cdot \psi^{*}\right) ; \psi^{*}\right) \\
& -\frac{1}{2 \pi} \int_{0}^{2 \pi} A^{4}\left(\phi-\phi^{*}\right)+A^{5} \nabla\left(\phi-\phi^{*}\right)+\operatorname{Re}\left(A^{6}\left(\psi-\psi^{*}\right)\right) d \theta \\
& +\tau-\frac{1}{2 \pi} \int_{0}^{2 \pi} \tau d \theta .
\end{aligned}
$$

Using the same method, we get

$$
\begin{aligned}
\Delta\left(\phi-\phi^{*}\right)= & A^{1}\left(\phi-\phi^{*}\right)+A^{2} \nabla\left(\phi-\phi^{*}\right)+\operatorname{Re}\left(A^{3}\left(\psi-\psi^{*}\right)\right) \\
& +\frac{1}{2 \pi} \int_{0}^{2 \pi} A^{4}\left(\phi-\phi^{*}\right)+A^{5} \nabla\left(\phi-\phi^{*}\right)+\operatorname{Re}\left(A^{6}\left(\psi-\psi^{*}\right)\right) d \theta \\
& +\tau-\frac{1}{2 \pi} \int_{0}^{2 \pi} \tau d \theta,
\end{aligned}
$$

and

$$
\begin{aligned}
\not\left(\psi-\psi^{*}\right)= & B^{1}\left(\phi-\phi^{*}\right)+B^{2} \nabla\left(\phi-\phi^{*}\right)+B^{3}\left(\psi-\psi^{*}\right) \\
& +\frac{1}{2 \pi} \int_{0}^{2 \pi} B^{4}\left(\phi-\phi^{*}\right)+B^{5} \nabla\left(\phi-\phi^{*}\right)+B^{6}\left(\psi-\psi^{*}\right) d \theta \\
& +h-\frac{1}{2 \pi} \int_{0}^{2 \pi} h d \theta
\end{aligned}
$$

where $A^{i}, B^{i}, i=1, \ldots, 6$ satisfy

$$
\begin{aligned}
& \left|A^{1}\right|+\left|A^{4}\right| \leq C(N)\left(|d \phi|^{2}+|d \phi||\psi|^{2}\right), \\
& \left|A^{2}\right|+\left|A^{5}\right|+\left|B^{3}\right|+|B|^{6} \leq C(N)\left(|d \phi|+|\psi|^{2}\right), \\
& \left|A^{3}\right|+\left|A^{6}\right|+\left|B^{1}\right|+\left|B^{4}\right| \leq C(N)|d \phi||\psi|, \\
& \left|B^{2}\right|+\left|B^{5}\right| \leq C(N)|\psi| .
\end{aligned}
$$


and

$$
\begin{aligned}
& \left\||x|^{\frac{3}{2}}\left(\left|A^{1}\right|+\left|A^{4}\right|\right)\right\|_{L^{4}\left(D_{2 r} \backslash D_{r}\right)} \\
& \quad+\left\||x|\left(\left|A^{3}\right|+\left|A^{6}\right|+\left|B^{1}\right|+\left|B^{4}\right|\right)\right\|_{L^{4}\left(D_{2 r} \backslash D_{r}\right)} \\
& \quad+\left\||x|^{\frac{1}{2}}\left(\left|A^{2}\right|+\left|A^{5}\right|+\left|B^{3}\right|+\left|B^{6}\right|\right)\right\|_{L^{4}\left(D_{2 r} \backslash D_{r}\right)} \\
& \quad+\left\|\left|B^{2}\right|+\left|B^{5}\right|\right\|_{L^{4}\left(D_{2 r} \backslash D_{r}\right)} \leq C \epsilon
\end{aligned}
$$

for any $t \in\left(\frac{1}{2} r_{n} R, 2 \delta\right)$ by (4.12).

Without loss of generality, we may assume $\delta=e^{m_{n} L} r_{n} R$ for some positive integer $m_{n}$ which tends to $\infty$ as $n \rightarrow \infty$. Substituting $u=\phi-\phi^{*}$ and $v=\psi-\psi^{*}$ in Corollary 3.5, we obtain the energy decay in the $\theta$-direction,

$$
\begin{aligned}
& \left\|r^{-1} \frac{\partial \phi}{\partial \theta}\right\|_{L^{2}\left(P_{i}\right)}+\left\|r^{-1} \frac{\partial \psi}{\partial \theta}\right\|_{L^{\frac{4}{3}\left(P_{i}\right)}} \leq\|\nabla u\|_{L^{2}\left(P_{i}\right)}+\|\nabla v\|_{L^{\frac{4}{3}}\left(P_{i}\right)} \\
& \leq C\left(\left(e^{i L} r_{n} R\right)^{\frac{1}{2}}+F_{0}^{1 / 2}(u, v)+F_{m_{n}}^{1 / 2}(u, v)\right) \\
& \left(e^{-\frac{1}{2}\left(m_{n}-i\right) L}+e^{-\frac{1}{2} i L}\right) \\
& \leq C(\sqrt{\epsilon}+\sqrt{\delta})\left(e^{-\frac{1}{2}\left(m_{n}-i\right) L}+e^{-\frac{1}{2} i L}\right),
\end{aligned}
$$

where the last inequality follows from Poincaré's inequality and the assumption (4.9).

By Corollary 2.4, we get

$$
\begin{aligned}
\left\|\frac{\partial \phi}{\partial r}\right\|_{L^{2}\left(P_{i}\right)} & \leq\left\|r^{-1} \frac{\partial \phi}{\partial \theta}\right\|_{L^{2}\left(P_{i}\right)}^{\frac{1}{2}}+C\left\|r^{-1} \frac{\partial \psi}{\partial \theta}\right\|_{L^{\frac{4}{3}}\left(P_{i}\right)}^{\frac{1}{2}}+C \sqrt{e^{i L} r_{n} R} \\
& \leq C\left(\epsilon^{\frac{1}{4}}+\delta^{\frac{1}{4}}\right)\left(e^{-\frac{1}{4}\left(m_{n}-i\right) L}+e^{-\frac{1}{4} i L}\right) .
\end{aligned}
$$

Therefore,

$$
\|\nabla \phi\|_{L^{2}\left(P_{i}\right)} \leq C\left(\epsilon^{\frac{1}{4}}+\delta^{\frac{1}{4}}\right)\left(e^{-\frac{1}{4}\left(m_{n}-i\right) L}+e^{-\frac{1}{4} i L}\right) .
$$

Then, by Theorem 2.1, we have

$$
E\left(\phi ; D_{\delta}\left(x_{n}\right) \backslash D_{r_{n} R}\left(x_{n}\right)\right) \leq \sum_{i=0}^{m_{n}-1}\|\nabla \phi\|_{L^{2}\left(P_{i}\right)}^{2} \leq C\left(\epsilon^{\frac{1}{2}}+\delta^{\frac{1}{2}}\right)
$$

and

$$
\begin{aligned}
O S c_{D_{\delta}\left(x_{n}\right) \backslash D_{r_{n} R}\left(x_{n}\right)} \phi_{n} & \leq C \sum_{i=0}^{m_{n}-1}\left(\|\nabla \phi\|_{L^{2}\left(P_{i}\right)}+e^{i L} r_{n} R\|\tau\|_{L^{2}\left(P_{i}\right)}\right) \\
& \leq C\left(\epsilon^{\frac{1}{4}}+\delta^{\frac{1}{4}}\right) .
\end{aligned}
$$

So, we have proved (4.1) and (4.3).

Combining this with equation (1.8), we get

$$
\begin{aligned}
\left\|\frac{\partial \psi}{\partial r}\right\|_{L^{\frac{4}{3}\left(P_{i}\right)}} & \leq\left\|r^{-1} \frac{\partial \psi}{\partial \theta}\right\|_{L^{\frac{4}{3}\left(P_{i}\right)}}+C\|\nabla \phi\|_{L^{2}\left(P_{i}\right)}\|\psi\|_{L^{4}\left(P_{i}\right)}+\|h\|_{L^{\frac{4}{3}\left(P_{i}\right)}} \\
& \leq C\left(\epsilon^{\frac{1}{4}}+\delta^{\frac{1}{4}}\right)\left(e^{-\frac{1}{4}\left(m_{n}-i\right) L}+e^{-\frac{1}{4} i L}\right)+C e^{i L} r_{n} R\|h\|_{L^{4}\left(P_{i}\right)}
\end{aligned}
$$




$$
\leq C\left(\epsilon^{\frac{1}{4}}+\delta^{\frac{1}{4}}\right)\left(e^{-\frac{1}{4}\left(m_{n}-i\right) L}+e^{-\frac{1}{4} i L}\right) .
$$

Thus,

$$
\|\nabla \psi\|_{L^{\frac{4}{3}}\left(P_{i}\right)} \leq C\left(\epsilon^{\frac{1}{4}}+\delta^{\frac{1}{4}}\right)\left(e^{-\frac{1}{4}\left(m_{n}-i\right) L}+e^{-\frac{1}{4} i L}\right) .
$$

Taking a cut-off function $\eta \in C_{0}^{\infty}\left(D_{\delta}\right)$, such that $0 \leq \eta \leq 1, \eta \equiv 1$ in $D_{\frac{1}{2} \delta} \backslash D_{2 r_{n} R}$ and

$$
|\nabla \eta| \leq \frac{C}{\delta} \text { in } D_{\delta} \backslash D_{\frac{1}{2} \delta} \text { and }|\nabla \eta| \leq \frac{C}{r_{n} R} \text { in } D_{2 r_{n} R} \backslash D_{r_{n} R},
$$

by the elliptic estimates for first order equations and Sobolev embedding, we obtain

$$
\begin{aligned}
\|\eta \psi\|_{L^{4}\left(D_{\delta}\right)} \leq & C\|\not \partial(\eta \psi)\|_{L^{\frac{4}{3}}\left(D_{\delta}\right)} \\
\leq & C\left(\frac{1}{\delta}\|\psi\|_{L^{\frac{4}{3}}\left(D_{\delta} \backslash D_{\frac{1}{2} \delta}\right)}+\frac{1}{r_{n} R}\|\psi\|_{L^{\frac{4}{3}}\left(D_{\left.2 r_{n} R \backslash D_{r_{n} R}\right)}\right.}\right. \\
& \left.+\|\nabla \psi\|_{L^{\frac{4}{3}}\left(D_{\delta} \backslash D_{r_{n} R}\right)}+\|h\|_{L^{\frac{4}{3}}\left(D_{\delta}\right)}\right) \\
\leq & C\left(\|\psi\|_{L^{4}\left(D_{\delta} \backslash D_{\frac{1}{2} \delta}\right)}+\|\psi\|_{L^{4}\left(D_{2 r_{n} R} \backslash D_{r_{n} R}\right)}\right. \\
& \left.+\sum_{i=1}^{m_{n}}\|\nabla \psi\|_{L^{\frac{4}{3}}\left(P_{i}\right)}+\delta\|h\|_{L^{4}\left(D_{\delta}\right)}\right) \\
\leq & C\left(\epsilon^{\frac{1}{4}}+\delta^{\frac{1}{4}}\right),
\end{aligned}
$$

where the last inequality follows from (4.22). This is

$$
E\left(\psi ; D_{\delta}\left(x_{n}\right) \backslash D_{r_{n} R}\left(x_{n}\right)\right) \leq C(\epsilon+\delta) .
$$

This is (4.2) and we finished the proof of Theorem 4.1.

Proof of Theorem 1.2 It is easy to see that Theorem 1.2 is a consequence of Theorem 4.1, the removable singularity Theorem 2.5 and the standard argument in [7].

Acknowledgements Open access funding provided by Max Planck Society.

Open Access This article is distributed under the terms of the Creative Commons Attribution 4.0 International License (http://creativecommons.org/licenses/by/4.0/), which permits unrestricted use, distribution, and reproduction in any medium, provided you give appropriate credit to the original author(s) and the source, provide a link to the Creative Commons license, and indicate if changes were made.

\section{References}

1. Branding, V.: On the evolution of regularized Dirac-harmonic maps from closed surfaces, arXiv:1406.6274

2. Chen, Q., Jost, J., Li, J., Wang, G.: Regularity theorems and energy identities for Dirac-harmonic maps. Mathematische Zeitschrift 251(1), 61-84 (2005)

3. Chen, Q., Jost, J., Li, J., Wang, G.: Dirac-harmonic maps. Mathematische Zeitschrift 254(2), 409-432 (2006)

4. Chen, Q., Jost, J., Sun, L., Zhu, M.: Estimates for solutions of Dirac equations and an application to a geometric elliptic-parabolic problem. J. Eur. Math. Soc. 79/2014 (Preprint)

5. Chen, Q., Jost, J., Wang, G., Zhu, M.: The boundary value problem for Dirac-harmonic maps. J. Eur. Math. Soc. (JEMS) 15(3), 997-1031 (2013) 
6. Deligne, P.: Quantum Fields and Strings: A Course for Mathematicians, vol. 2. Amer Mathematical Society, New York (1999)

7. Ding, W., Tian, G.: Energy identity for a class of approximate harmonic maps from surfaces. Comm. Anal. Geom. 3(3-4), 543-554 (1995)

8. Gilbarg, D., Trudinger, N.S.: Elliptic Partial Differential Equations of Second Order. Springer Verlag, Heidelberg (2001)

9. Jost, J.: Two-Dimensional Geometric Variational Problems. Wiley, New York (1991)

10. Jost, J.: Geometry and Physics. Springer, Berlin (2009)

11. Jost, J., Liu, L., Zhu, M.: Geometric analysis of the action functional of the nonlinear supersymmetric sigma model, MPI MIS Preprint: 77/2015

12. Jost, J., Liu, L., Zhu, M.: A global weak solution of the Dirac-harmonic map flow, to appear in Ann. Inst. H. Poincare Anal. Non Lineaire (2017). doi:10.1016/j.anihpc.2017.01.002

13. Jost, J., Liu, L., Zhu, M.: Energy identity for a class of approximate Dirac-harmonic maps from surfaces with boundary, MPI MIS Preprint: 11/2017

14. Lawson, H., Michelsohn, M.: Spin Geometry, vol. 38. Princeton University Press, Princeton (1989)

15. Li, J., Zhu, X.: Energy identity for the maps from a surface with tension field bounded in $L^{p}$. Pacific J. Math. 260(1), 181-195 (2012)

16. Li, Y., Wang, Y.: A weak energy identity and the length of necks for a sequence of Sacks-Uhlenbeck -harmonic maps. Adv. Math. 225(3), 1134-1184 (2010)

17. Lin, F., Wang, C.: Energy identity of harmonic map flow from surfaces at finite singular time. Calc. Var. Partial Differ. Equ. 6, 369-380 (1998)

18. Liu, L.: No neck for Dirac-harmonic maps. Calcul. Var. Partial Differ. Equ. 52(1-2), 1-15 (2015)

19. Liu, L., Yin, H.: Neck analysis for biharmonic maps. Mathematische Zeitschrift 283(3-4), 807-834 (2016)

20. Liu, L., Yin, H.: On the finite time blow-up of biharmonic map flow in dimension four. J. Elliptic Parabolic Equ. 1, 363-385 (2015)

21. Parker, T.: Bubble tree convergence for harmonic maps. J. Diff. Geom. 44(3), 595-633 (1996)

22. Qing, J., Tian, G.: Bubbling of the heat flows for harmonic maps from surfaces. Commun. Pure Appl. Math. 50(4), 295-310 (1997)

23. Sacks, J., Uhlenbeck, K.: The existence of minimal immersions of 2-spheres. Ann. Math. 113, 1-24 (1981)

24. Sharp, B., Zhu, M.: Regularity at the free boundary for Dirac-harmonic maps from surfaces. Calc. Var. Partial Differ. Equ. 55(2), 55:27 (2016)

25. Simon, L.: Asympotics for a class of nolinear evolution equations with applications to geometric problems. Ann. Math. 118, 525-571 (1983)

26. Sharp, B., Topping, P.: Decay estimates for Rivières equation, with applications to regularity and compactness. Trans. Am. Mathe. Soc. 365(5), 2317-2339 (2013)

27. Rivière, T.: Conservation laws for conformally invariant variational problems. Invent. Math. 168, 1-22 (2007)

28. Rivière, T.: Conformally Invariant 2-dimensional Variational Problems. Cours joint de IInstitut Henri Poincaré, Paris XII Creteil, November (2010)

29. Wang, C.: Remarks on approximate harmonic maps in dimension two. Calc. Var. Partial Differ. Equ. 56:23(2), 56 (2017). 24 pp

30. Wang, C., Xu, D.: Regularity of Dirac-harmonic maps. Int. Math. Res. Not. IMRN 20, 3759-3792 (2009)

31. Wang, W., Wei, D., Zhang, Z.: Energy identity for approximate harmonic maps from surface to general targets. J. Funct. Anal. 272(2), 776-803 (2017)

32. Zhao, L.: Energy identities for Dirac-harmonic maps. Calcul. Var. Partial Differ. Equ. 28(1), 121-138 (2007)

33. Zhu, M.: Regularity of weakly Dirac-harmonic maps to hypersurfaces. Ann. Global Anal. Geom. 35(4), 405-412 (2009) 